LA WRENCE LIWEAMORE NATIONAL LABDPATOAY

\title{
Radial Reflection Diffraction Tomography
}

S. K. Lehman, S. J. Norton

November 03, 2003 


\section{Disclaimer}

This document was prepared as an account of work sponsored by an agency of the United States Government. Neither the United States Government nor the University of California nor any of their employees, makes any warranty, express or implied, or assumes any legal liability or responsibility for the accuracy, completeness, or usefulness of any information, apparatus,

product, or process disclosed, or represents that its use would not infringe privately owned rights. Reference herein to any specific commercial product, process, or service by trade name, trademark, manufacturer, or otherwise, does not necessarily constitute or imply its endorsement, recommendation, or favoring by the United States Government or the University of California. The views and opinions of authors expressed herein do not necessarily state or reflect those of the United States Government or the University of California, and shall not be used for advertising or product endorsement purposes.

\section{Auspices Statement}

This work was performed under the auspices of the U.S. Department of Energy by University of California, Lawrence Livermore National Laboratory under Contract W-7405-Eng-48. 


\title{
Radial Reflection Diffraction Tomography
}

\author{
Sean K. Lehman ${ }^{*}$ Stephen J. Norton ${ }^{\dagger}$
}

November 7, 2003

UCRL-TR-200707

${ }^{*}$ Lawrence Livermore National Laboratory, L-154, 7000 East Avenue, Livermore, CA 94566, lehman2@llnl.gov

${ }^{\dagger}$ Geophex, Ltd., 605 Mercury Street, Raleigh, NC 27603 


\begin{abstract}
We develop a wave-based tomographic imaging algorithm based upon a single rotating radially outward oriented transducer. At successive angular locations at a fixed radius, the transducer launches a primary field and collects the backscattered field in a "pitch/catch" operation. The hardware configuration, operating mode, and data collection method is identical to that of most medical intravascular ultrasound (IVUS) systems. IVUS systems form images of the medium surrounding the probe based upon ultrasonic B-scans, using a straight-ray model of sound propagation. Our goal is to develop a wave-based imaging algorithm using diffraction tomography techniques. Given the hardware configuration and the imaging method, we refer to this system as "radial reflection diffraction tomography." We consider two hardware configurations: a multimonostatic mode using a single transducer as described above, and a multistatic mode consisting of a single transmitter and an aperture formed by multiple receivers. In this latter case, the entire source/receiver aperture rotates about the fixed radius. Practically, such a probe is mounted at the end of a catheter or snaking tube that can be inserted into a part or medium with the goal of forming images of the plane perpendicular to the axis of rotation. We derive an analytic expression for the multimonostatic inverse but ultimately use the new Hilbert space inverse wave (HSIW) algorithm to construct images using both operating modes. Applications include improved IVUS imaging, bore hole tomography, and non-destructive evaluation (NDE) of parts with existing access holes.
\end{abstract}




\section{Introduction}

Consider a wave-based tomographic imaging tool consisting of a single transducer rotating about a fixed center. The transducer is oriented such that it launches fields radially outward. At each angular location, the transducer launches a primary field and collects the backscattered field in a "pitch/catch" operation. This configuration, in which a single transducer acts as both source and receiver at multiple spatial locations, is known as multimonostatic. The configuration is shown in Figure 1(a). One may also consider an annular array of fixed transducers. In succession, each transducer launches a primary field and the backscattered field is measured on all the transducers. This configuration, with multiple spatially diverse transmitters and receivers, is a multistatic operating mode and is shown in Figure 1(b). A second multistatic configuration, presented in Figure 1(c), consists of a rotating sub-aperture formed by a single transmitter surrounded by multiple receivers. At each angular location, the transmitter launches the primary field and the backscattered field is measured on all receivers.

The goal of this tool is to use inverse wave techniques to reconstruct, that is, to form images, of the medium surrounding the probe, in the plane perpendicular to the axis of rotation. Given the arrangement of transducer(s) at a fixed radius collecting reflected scattered fields, we refer to this as a radial reflection configuration. We use the diffraction tomography[1,2] technique, based upon a linearized scattering model, to form images. Thus, given the physical transducer configuration and the mathematical method used to invert the scattering, we call the algorithm radial reflection diffraction tomography (RRDT).

When operating in a multimonostatic reflection mode, a spectrally wide band, that is a frequency diverse, incident source must be used because there is insufficient spatial diversity to form images of the surrounding medium. The planar reconstruction has two spatial variables, by the "Golden Rule" of tomography[3], the measurement system must then have at least two free parameters. Angular location is one and incident source frequency is the other. Conceptually, the forward scattering process maps the two spatial variables of a physical object into the angular location and frequency parameters of the measured field.

Practically, such a probe is mounted at the end of a catheter or snaking tube that can be inserted into a part or medium with the purpose of forming images of the plane perpendicular to the axis of rotation. Applications include intravascular ultrasound (IVUS)[4], bore hole tomography, and nondestructive evaluation (NDE) of parts with existing access holes. We seek a wave-based imaging algorithm rather than a straight-ray B-scan [2] algorithm which is used, for example, in IVUS systems. A wave-based inversion more accurately describes the physics of wave field propagation.

In the next section, we develop the forward linearized multimonostatic scattering model. We initially develop a three-dimensional model. As we wish, however, to form images in a plane normal to the probe axis, we specialize the model to 2.5-dimensions assuming no variation in $z$, that is, along the axis of the catheter. In Section 3, we develop the RRDT Fourier Diffraction Theorem [1, 2] (FDT) which governs how the medium's spatial spectrum is sampled by the forward measurement process. The RRDT FDT dictates the resolution of the reconstruction. We develop an analytic expression for multimonostatic inversion in Section 4. This inverse, however, proved impractical to implement numerically. In its place, we use the new Hilbert space inverse 
wave (HSIW) algorithm [5] outlined in Section 5 to achieve imaging algorithms not only for the multimonostatic configuration but also for the multistatic configurations.

Due to the heavy computation nature of the HSIW, a trade-off must be made between the number of transducers and frequencies used in the reconstruction, and the quality of the reconstructed image. Ideally, an aperture consisting of a 360 degree annular array would yield the best image. Practically, it is currently not possible to construct such a fully wired array in, for example, a 0.25 mm IVUS probe. Thus, we study rotating sub-apertures such as presented in Figure 1 (c), and compare reconstruction results to the single multimonostatic configuration.

As explained above, when operating in a reflection mode, the imaging mathematics dictate that spectrally wide band pulses must be used. The range resolution of the reconstructed image is proportional to the number of frequencies used in the reconstruction. Under the Hilbert space inverse wave algorithm, however, increasing the number of frequencies and transducers, increases the complexity of the reconstruction, the size of the intermediary data files, reconstruction time, and computer memory requirement. Thus, the trade-off between computer resources and resolution must also be considered.

We initially study these issues in the proof-of-principle presented in Section 6 where we reconstruct scattered fields obtained from a simulation based upon the linearized forward scattering model of Section 2. The simulated medium consists of a collection of point scatterers. We compare the multistatic and multimonostatic configurations. In Section 7, we provide a more rigorous test of the HSIW algorithm by inverting data obtained from Lawrence Livermore National Laboratory's acoustic/elastic finite-difference time-domain propagation and scattering code, E3D $[6,7,8,9,10,11]$. We simulated a medium with two scattering objects whose physical properties varied from 5\% below the background to $15 \%$ above it. Again, we compare multimonostatic and multistatic configurations. Conclusions are presented in Section 8.

\section{Multimonostatic Forward Scattering Model}

We develop here the linearized forward scattering model derivation for the multimonostatic configuration. We are interested in imaging an area surrounding the probe in the plane normal to the axis of rotation. The geometry is that of Figure 2. The transducer location is specified by $\mathbf{r}_{0} \equiv R_{0}\left(\cos \theta_{0}, \sin \theta_{0}\right)$ where $R_{0}$ is the probe radius, a constant. At each angular location, $\theta_{0}$, the transducer launches the primary field radially outward into the medium and measures the reflected scattered field. The wave propagation and scattering is governed by the Helmholtz equation,

$$
\left[\nabla^{2}+k^{2}(\mathbf{r})\right] \psi(\mathbf{r}, \omega)=-p(\mathbf{r}, \omega),
$$

where $\mathbf{r} \equiv r(\cos \theta, \sin \theta)$ is the spatial coordinate of a point in the surrounding medium, $\omega$ is the temporal frequency, $k(\mathbf{r})$ is the wavenumber of the medium, $\psi(\mathbf{r}, \omega)$ is the total field, and $p(\mathbf{r}, \omega)$ is the incident pulse temporal spectrum.

The derivation is simplified by rearranging Eqn. 1 to remove the spatial inhomogeneity in the scattering operator in the left hand side. We add the background wavenumber, $k_{0}(\omega) \equiv \omega / v_{0}$, to 
both sides of Eqn. 1 and move the inhomogeneous term to the right hand side to obtain,

$$
\left[\nabla^{2}+k_{0}^{2}\right] \psi(\mathbf{r}, \omega)=-p(\mathbf{r}, \omega)-\left[k^{2}(\mathbf{r})-k_{0}^{2}(\omega)\right] \psi(\mathbf{r}, \omega) .
$$

Define the object function as

$$
o(\mathbf{r}) \equiv \frac{k^{2}(\mathbf{r})}{k_{0}^{2}}-1
$$

and express Eqn. 2 as

$$
\left[\nabla^{2}+k_{0}^{2}\right] \psi(\mathbf{r}, \omega)=-p(\mathbf{r}, \omega)-k_{0}^{2}(\omega) o(\mathbf{r}) \psi(\mathbf{r}, \omega) .
$$

The second term on the right hand side is known as the secondary source which creates the scattered field. We may use Green's theorem[12] to cast the differential equation of Eqn. 4 into an integral equation via

$$
\psi(\mathbf{R}, \omega)=\int d \mathbf{r} G(\mathbf{R}, \mathbf{r}, \omega) p(\mathbf{r}, \omega)+k_{0}^{2}(\omega) \int d \mathbf{r} G(\mathbf{R}, \mathbf{r}, \omega) o(\mathbf{r}) \psi(\mathbf{r}),
$$

where the Green function is

$$
G(\mathbf{R}, \mathbf{r}, \omega)=\frac{e^{i k_{0}(\omega)|\mathbf{R}-\mathbf{r}|}}{4 \pi|\mathbf{R}-\mathbf{r}|}
$$

The first integral in Eqn. 5 is the primary field, $\psi^{\text {inc }}(\mathbf{R}, \omega)$. Subtracting it from the total field yields the scattered field,

$$
\psi^{\text {scatt }}(\mathbf{R}, \omega) \equiv \psi(\mathbf{R}, \omega)-\psi^{i n c}(\mathbf{R}, \omega)=k_{0}^{2}(\omega) \int d \mathbf{r} G(\mathbf{R}, \mathbf{r}, \omega) o(\mathbf{r}) \psi(\mathbf{r}, \omega) .
$$

An expression for the measured scattered field is obtained when $\psi^{\text {scatt }}(\mathbf{R}, \omega)$ is evaluated on the measurement surface, $\mathbf{r}_{0}$,

$$
\psi^{\text {scatt }}\left(\mathbf{r}_{0}, \omega\right)=k_{0}^{2}(\omega) \int d \mathbf{r} G\left(\mathbf{r}_{0}, \mathbf{r}, \omega\right) o(\mathbf{r}) \psi(\mathbf{r}, \omega) .
$$

Given the scattered field, the data, on the measurement surface, $\mathbf{r}_{0}=\left(R_{0}, \theta_{0}\right) \forall \theta_{0}$, we wish to invert Eqn. 7 to reconstruct an estimate of the scattering medium, $o(\mathbf{r})$, in the plane perpendicular to the axis of transducer rotation. The equation is nonlinear in that the total field which is the sum of the incident and scattered fields, appears under the integral. We may simplify this if we assume the medium is weakly scattering and that the first Born approximation holds. We then neglect the scattered field term and express Eqn. 7 as

$$
\psi_{B}^{\text {scatt }}\left(\mathbf{r}_{0}, \omega\right)=k_{0}^{2}(\omega) \int d \mathbf{r} G\left(\mathbf{r}_{0}, \mathbf{r}, \omega\right) o(\mathbf{r}) \psi^{\text {inc }}(\mathbf{r}, \omega),
$$

where we use the $B$ subscript to indicate the Born approximation has been used. We further assume the incident field is the result of a point source located at $\mathbf{r}_{0}$, so that $p(\mathbf{r}, \omega)=P(\omega) \delta\left(\mathbf{r}_{0}-\mathbf{r}\right)$, 
where $P(\omega)$ is the incident pulse spectrum. We model neither antenna characteristics nor beam pattern. With this assumption, the incident field is,

$$
\psi^{\text {inc }}(\mathbf{r}, \omega)=P(\omega) G\left(\mathbf{r}_{0}, \mathbf{r}, \omega\right),
$$

and Eqn. 8 reads

$$
\psi_{B}^{\text {scatt }}\left(\mathbf{r}_{0}, \omega\right)=P(\omega) k_{0}^{2}(\omega) \int d \mathbf{r} G^{2}\left(\mathbf{r}_{0}, \mathbf{r}, \omega\right) o(\mathbf{r})
$$

where the squared Green function is a result of the transmitter and receiver being co-located. Using Eqn. 6, we explicitly express the forward scattering model as

$$
\psi_{B}^{\text {scatt }}\left(\mathbf{r}_{0}, \omega\right)=\frac{P(\omega) k_{0}^{2}(\omega)}{(4 \pi)^{2}} \int d \mathbf{r} \frac{e^{i 2 k_{0}(\omega)\left|\mathbf{r}_{0}-\mathbf{r}\right|}}{\left|\mathbf{r}_{0}-\mathbf{r}\right|^{2}} o(\mathbf{r})
$$

The squared Green function is problematic in the development of an inverse scattering expression since the diffraction tomography technique requires an expansion of the integral kernel in a series along the measurement surface. We simplify Eqn. 10 following a technique used by Norton and Linzer[13]. We first define the weighted scattered field as

$$
\psi_{W}^{\text {scatt }}\left(\mathbf{r}_{0}, 2 \omega\right) \equiv \frac{4 \pi}{P(\omega) k_{0}^{2}(\omega)} \psi_{B}^{\text {scatt }}\left(\mathbf{r}_{0}, \omega\right)=\frac{1}{4 \pi} \int d \mathbf{r} \frac{e^{i 2 k_{0}(\omega)\left|\mathbf{r}_{0}-\mathbf{r}\right|}}{\left|\mathbf{r}_{0}-\mathbf{r}\right|^{2}} o(\mathbf{r}),
$$

and then differentiate it with respect to $k_{0}(\omega)$,

$$
\begin{aligned}
\frac{d}{d k_{0}(\omega)} \psi_{W}^{\text {scatt }}\left(\mathbf{r}_{0}, 2 \omega\right) & =\frac{i 2}{4 \pi} \int d \mathbf{r} \frac{e^{i 2 k_{0}(\omega)\left|\mathbf{r}_{0}-\mathbf{r}\right|}}{\left|\mathbf{r}_{0}-\mathbf{r}\right|} o(\mathbf{r}) \\
& =i 2 \int d \mathbf{r} G\left(\mathbf{r}_{0}, \mathbf{r}, 2 \omega\right) o(\mathbf{r})
\end{aligned}
$$

For notational convenience, we define

$$
\phi\left(\mathbf{r}_{0}, 2 \omega\right) \equiv \frac{d}{d k_{0}(\omega)} \psi_{W}^{\text {scatt }}\left(\mathbf{r}_{0}, 2 \omega\right)=v_{0} \frac{d}{d \omega} \psi_{W}^{\text {scatt }}\left(\mathbf{r}_{0}, 2 \omega\right)
$$

and express Eqn. 12 as

$$
\phi\left(\mathbf{r}_{0}, 2 \omega\right)=i 2 \int d \mathbf{r} G\left(\mathbf{r}_{0}, \mathbf{r}, 2 \omega\right) o(\mathbf{r})
$$

Eqn. 14 is our forward scattering model in three dimensions. Since our goal is to invert this equation to reconstruct the medium in a plane perpendicular to the axis of rotation, we simplify this further, in the next section, by assuming the object function, $o(\mathbf{r})$, is independent of the vertical $(z)$ coordinate. This is known as a "2.5-dimensional" problem. 


\section{$2.1 \quad$ 2.5-Dimensional Problem}

In cylindrical coordinates, $\mathbf{r} \equiv(r, \theta, z)$ and the measurement surface is $\mathbf{r}_{0} \equiv\left(R_{0}, \theta_{0}, z_{0}\right)$ for $R_{0}$ fixed and $0 \leq \theta_{0}<2 \pi$. Thus the volume integral of Eqn. 14 becomes

$$
\phi\left(\mathbf{r}_{0}, 2 \omega\right)=i 2 \int_{0}^{\infty} r d r \int_{0}^{2 \pi} d \theta o(r, \theta) \int_{-\infty}^{\infty} d z G\left(\mathbf{r}_{0}, \mathbf{r}, 2 \omega\right) .
$$

The distance between the observation point, $\mathbf{r}_{0}$, and the scattering point is given by

$$
\left|\mathbf{r}_{0}-\mathbf{r}\right|^{2}=R^{2}+\left(z_{0}-z\right)^{2}
$$

where the planar component of the distance is

$$
R^{2} \equiv R_{0}^{2}+r^{2}-2 R_{0} r \cos \left(\theta_{0}-\theta\right)
$$

With this definition, we note that the $z$-integral of the Green function reduces to

$$
\int_{-\infty}^{\infty} d z G\left(\mathbf{r}_{0}, \mathbf{r}, 2 \omega\right)=\frac{i}{4} H_{0}^{(1)}\left(2 k_{0}(\omega) R\right)
$$

so that Eqn. 15 reads

$$
\phi\left(\mathbf{r}_{0}, 2 \omega\right)=-\frac{1}{2} \int_{0}^{\infty} r d r \int_{0}^{2 \pi} d \theta o(r, \theta) H_{0}^{(1)}\left(2 k_{0}(\omega) R\right)
$$

This is the 2.5-dimensional forward scattering model. It can be interpreted as a mapping from the object's two-dimensional $(r, \theta)$ space into the measured data's two-dimensional $\left(\theta_{0}, \omega\right)$ measurement space. In the next section, we develop the equivalent of the Fourier Diffraction Theorem $[1,2]$ (FDT) for this measurement system. The FDT determines the spatial resolution of the reconstruction by showing what part of the object's spatial spectrum is sampled by the measurement system.

\section{Fourier Diffraction Theorem}

The Fourier Diffraction Theorem (FDT) relates the one-dimensional spectrum of the measured data along the measurement surface, to the planar (2-dimensional) spatial spectrum of the object function. We begin the derivation by replacing the Hankel function in Eqn. 16 with the expansion,

$$
H_{0}^{(1)}\left(k_{0}(\omega) R\right)=\sum_{n=-\infty}^{\infty} J_{n}\left(k_{0}(\omega) R_{0}\right) H_{n}^{(1)}\left(k_{0}(\omega) r\right) e^{i n\left(\theta_{0}-\theta\right)}
$$

where $R_{0}<r$. Substituting this into Eqn. 16 yields

$$
\phi\left(\mathbf{r}_{0}, 2 \omega\right)=-\frac{1}{2} \sum_{n=-\infty}^{\infty} \int_{0}^{\infty} r d r \int_{0}^{2 \pi} d \theta o(r, \theta) J_{n}\left(2 k_{0}(\omega) R_{0}\right) H_{n}^{(1)}\left(2 k_{0}(\omega) r\right) e^{i n\left(\theta_{0}-\theta\right)} .
$$


We now Fourier expand the object and measured field functions along the measurement surface using following transform pairs,

$$
\begin{aligned}
o_{n}(r) & =\frac{1}{2 \pi} \int_{0}^{2 \pi} d \theta o(r, \theta) e^{-i n \theta} \\
o(r, \theta) & =\sum_{n=-\infty}^{\infty} o_{n}(r) e^{i n \theta}
\end{aligned}
$$

and

$$
\begin{aligned}
\phi_{n}(2 \omega) & =\frac{1}{2 \pi} \int_{0}^{2 \pi} d \theta_{0} \phi\left(\mathbf{r}_{0}, 2 \omega\right) e^{-i n \theta_{0}} \\
\phi\left(\mathbf{r}_{0}, 2 \omega\right) & =\sum_{n=-\infty}^{\infty} \phi_{n}(2 \omega) e^{i n \theta_{0}}
\end{aligned}
$$

Using Eqn. 20 to transform Eqn. 17, yields

$$
\begin{aligned}
\frac{1}{2 \pi} \int_{0}^{2 \pi} d \theta_{0} \phi\left(\mathbf{r}_{0}, 2 \omega\right) e^{-i m \theta_{0}=} & -\frac{1}{4 \pi} \sum_{n=-\infty}^{\infty} J_{n}\left(2 k_{0}(\omega) R_{0}\right) \int_{0}^{\infty} r d r H_{n}^{(1)}\left(2 k_{0}(\omega) r\right) \times \\
& \int_{0}^{2 \pi} d \theta o(r, \theta) e^{-i n \theta} \int_{0}^{2 \pi} d \theta_{0} e^{i(n-m) \theta_{0}} .
\end{aligned}
$$

We use Eqn. 18 and

$$
\frac{1}{2 \pi} \int_{0}^{2 \pi} d \theta_{0} e^{i(m-n) \theta_{0}}=\delta_{m n}
$$

to reduce Eqn. 22 to

$$
\phi_{m}(2 \omega)=-\pi J_{m}\left(2 k_{0}(\omega) R_{0}\right) \int_{0}^{\infty} r d r H_{m}^{(1)}\left(2 k_{0}(\omega) r\right) o_{m}(r)
$$

Inverting Eqn. 23 to obtain the transform of the object function, $o_{m}(\mathbf{r})$, is not possible because no orthogonality relation exists for Hankel functions. If we make the assumption, however, that the object, $o(r, \theta)$, is real we have from Eqn. 18 that

$$
o_{-m}^{*}(r)=o_{m}(r) \text {. }
$$

Using this and the property of Hankel functions,

$$
(-1)^{m} H_{-m}^{(1)}\left(2 k_{0}(\omega) r\right)=H_{m}^{(1)}\left(2 k_{0}(\omega) r\right)
$$

we may solve for $o_{m}(r)$ by observing that

$$
\phi_{m}(2 \omega)+(-1)^{m} \phi_{-m}^{*}(2 \omega)=-2 \pi J_{m}\left(2 k_{0}(\omega) R_{0}\right) \int_{0}^{\infty} r d r J_{m}\left(2 k_{0}(\omega) r\right) o_{m}(r),
$$


results in a Bessel transform of the object,

$$
o_{m}\left(2 k_{0}(\omega)\right) \equiv \int_{0}^{\infty} r d r J_{m}\left(2 k_{0}(\omega) r\right) o_{m}(r)
$$

which is invertible. Using Eqn. 25, we solve Eqn. 24 for the object Bessel transform,

$$
o_{m}\left(2 k_{0}(\omega)\right)=-\frac{1}{2 \pi J_{m}\left(2 k_{0}(\omega) R_{0}\right)}\left[\phi_{m}(2 \omega)+(-1)^{m} \phi_{-m}^{*}(2 \omega)\right] .
$$

Explicitly expressing Eqn. 26 in terms of the measured field, we obtain the "Fourier-Bessel Diffraction Theorem" for RRDT:

$$
o_{m}\left(2 k_{0}(\omega)\right)=-\frac{2 v_{0}^{3}}{J_{m}\left(2 k_{0}(\omega) R_{0}\right)} \frac{d}{d \omega}\left[\frac{1}{\omega^{2}}\left(\frac{\psi_{m}(\omega)}{P(\omega)}+\frac{\psi_{-m}^{*}(\omega)}{P^{*}(\omega)}\right)\right] .
$$

We believe it is more intuitive to relate the Fourier-Bessel transform of the object to its conventional two-dimensional Fourier transform. We do so in the next section.

\subsection{The RRDT Fourier Diffraction Theorem}

We wish to relate the Fourier-Bessel object transform of Eqn. 27 in the $(k, m)$ wavenumber/angular index space to a continuous variable wavenumber/angle Fourier space. The relation is developed in Appendix A. We substitute Eqn. 27 into Eqn. 66 of Appendix A, to obtain the Fourier Diffraction Theorem for RRDT,

$$
\begin{aligned}
O\left(2 k_{0}(\omega), \phi\right) & =\sum_{m=-\infty}^{\infty}(-i)^{m} e^{i m \phi} o_{m}\left(2 k_{0}(\omega)\right) \\
& =-2 v_{0}^{3} \sum_{m=-\infty}^{\infty} \frac{(-i)^{m} e^{i m \phi}}{J_{m}\left(2 k_{0}(\omega) R_{0}\right)} \frac{d}{d \omega}\left[\frac{1}{\omega^{2}}\left(\frac{\psi_{m}(\omega)}{P(\omega)}+\frac{\psi_{-m}^{*}(\omega)}{P^{*}(\omega)}\right)\right],
\end{aligned}
$$

where the Fourier space polar pair $\left(2 k_{0}(\omega), \phi\right)$ are related to the Fourier space Cartesian pair $\left(k_{x}, K_{y}\right)$ via Eqn. 63.

This relationship shows that at each spatial spectrum angular location, $\phi$, the locus covered in the object's spatial spectrum is a radial line, $k_{0}(\omega)$, that extends over the temporal spectral bandwidth of the pulse. When all angular locations are combined, the area is a torus whose inner and outer radii are proportional to the lower and upper pulse spectrum cutoff frequencies, respectively. If, for example, the pulse spectrum has a lower cutoff of $\omega_{L}$ and an upper cutoff of $\omega_{U}$, the inner and outer radii of the torus are given by $2 k_{0}\left(\omega_{L}\right)$ and $2 k_{0}\left(\omega_{U}\right)$, respectively. This shown in Figure 3. The implication of this relationship is that radial resolution (alternatively, range resolution) is related to the spectral bandwidth of the incident field: the wider the bandwidth, the greater the resolution. 


\section{Analytic Multimonostatic Inverse}

We use the orthogonality of Bessel functions to invert Eqn. 24 to obtain the transform of the object function:

$$
o_{m}(r)=-\frac{1}{\pi} \int_{0}^{\infty} k_{0}(\omega) d k_{0}(\omega)\left[\frac{\phi_{m}(2 \omega)+(-1)^{m} \phi_{-m}^{*}(2 \omega)}{J_{m}\left(2 k_{0}(\omega) R_{0}\right)}\right] J_{m}\left(2 k_{0}(\omega) r\right) .
$$

Combining Eqns. 19 and 13 with Eqn. 29 yields the reconstruction:

$$
\hat{o}(r, \theta)=-8 v_{0} \sum_{m=-\infty}^{\infty} e^{i m \theta} \int_{0}^{\infty} \omega d \omega \frac{d}{d \omega}\left[\frac{1}{\omega^{2}}\left(\frac{\psi_{m}(\omega)}{P(\omega)}+\frac{\psi_{-m}^{*}(\omega)}{P^{*}(\omega)}\right)\right] \frac{J_{m}\left(2 k_{0}(\omega) r\right)}{J_{m}\left(2 k_{0}(\omega) R_{0}\right)}
$$

This analytic inversion has proved impractical to implement numerically. The reciprocal of the Bessel function results in poles along the real frequency axis obliging a contour integral. We were unsuccessful, however at identifying a contour in which every function within the integrand remained finite. This compelled us to seek a purely numerical solution to the inversion problem. We used the Hilbert space wave inversion algorithm developed by Devaney [5], and summarize it in the following section.

\section{Hilbert Space Wave Inversion}

The Hilbert space inverse wave (HSIW) algorithm permits us to develop an inverse for any geometry with any combination of sources, receivers, and frequencies. Because we have this flexibility, we develop the HSIW theory for the most general multistatic, wide band case where we have spatial diversity in both the sources and receivers, as well as frequency diversity. In an actual radial reflection device such as an intravascular ultrasound probe, the data are collected at discrete angular locations. We denote by

$$
\mathbf{R}_{n}^{t} \equiv R_{0}\left(\cos \theta_{n}, \sin \theta_{n}\right)
$$

the transmitter locations where $\theta_{n}=n \Delta \theta_{\text {src }}$ for $n=0,1, \cdots, N_{\text {src }}-1$ where $N_{\text {src }}=2 \pi / \Delta \theta$. Similarly, the receiver locations are given by

$$
\mathbf{R}_{m}^{r} \equiv R_{0}\left(\cos \theta_{m}, \sin \theta_{m}\right)
$$

where $\theta_{m}=m \Delta \theta_{\mathrm{rcv}}$ for $m=0,1, \cdots, N_{\mathrm{rcv}}-1$ where $N_{\mathrm{rcv}}=2 \pi / \Delta \theta_{\mathrm{rcv}}$.

For each source, the receiver(s) record the backscattered field as a time series that is digitized for processing. Fourier transforming the time series data results in the spectrum of the measured wave form at discrete frequencies. The forward scattering equation under the Born approximation with both spatial and frequency diversity is

$$
\psi_{B}^{s c a t}\left(\mathbf{R}_{m}^{r}, \mathbf{R}_{n}^{t}, \omega_{l}\right)=P\left(\omega_{l}\right) k_{0}^{2}\left(\omega_{l}\right) \int d \mathbf{r}^{\prime} G\left(\mathbf{R}_{m}^{r}, \mathbf{r}^{\prime}, \omega_{l}\right) o\left(\mathbf{r}^{\prime}\right) G\left(\mathbf{r}^{\prime}, \mathbf{R}_{n}^{t}, \omega_{l}\right),
$$


where $\omega_{l}, l=0,1, \cdots, N_{f}-1$ are the discrete frequencies and $N_{f}$ is the number of frequencies in the pulse band width. For the multimonostatic case of Eqn. 9, this reduces to

$$
\psi_{B}^{s c a t}\left(\mathbf{R}_{n}^{t}, \omega_{l}\right)=P\left(\omega_{l}\right) k_{0}^{2}\left(\omega_{l}\right) \int d \mathbf{r}^{\prime} G^{2}\left(\mathbf{R}_{n}^{t}, \mathbf{r}^{\prime}, \omega_{l}\right) o\left(\mathbf{r}^{\prime}\right)
$$

The HSIW interprets Eqn. 33 as a mapping from a continuous object space to a discrete measurement space. The object space is the physical $(x, y)$ space of the object function. The measurement space consists of the discrete angles and temporal frequencies at which the scattered data are collected. The scattering operator projects the object onto the measurement space. We define the forward propagation or projection kernel as

$$
\Pi^{*}(\mathbf{r}) \equiv P\left(\omega_{l}\right) k_{0}^{2}\left(\omega_{l}\right) G\left(\mathbf{R}_{m}^{r}, \mathbf{r}, \omega_{l}\right) G\left(\mathbf{r}, \mathbf{R}_{n}^{t}, \omega_{l}\right),
$$

where $\Pi(\mathbf{r})$ is a $J \equiv\left(N_{\text {src }} \times N_{\mathrm{rcv}} \times N_{f}\right)$ element column vector. Mathematically, the projection is represented as an inner product between the object function and the kernel via,

$$
D=\int d \mathbf{r} \Pi^{*}(\mathbf{r}) o(\mathbf{r}) \equiv\langle\Pi, o\rangle,
$$

where $D$ is a $J$ element column vector where each element represents a particular source, receiver, and frequency combination. Symbolically, we define the forward scattering operator, $K$, as

$$
K[\cdot] \equiv \int d \mathbf{r} \Pi^{*}(\mathbf{r})[\cdot]
$$

The HSIW is a method used to derive an inverse of this operator. Consider the singular value decomposition (SVD) of $K$ :

$$
K=U S V^{\dagger}
$$

where the columns of $U$ form an orthonormal set of column vectors, $u_{j}$, which span the measured data space, and the components of $V$ form an orthonormal set of vectors, $v_{j}(\mathbf{r})$, which span the object space. $S$ is a diagonal matrix of singular values, $\sigma_{j}$. We wish to emphasize that the $u_{j}$ are column vectors where as the $v_{j}(\mathbf{r})$ are complex functions of $\mathbf{r}$. The set of normal equations for this singular system are

$$
\begin{aligned}
K v_{j}(\mathbf{r}) & =\sigma_{j} u_{j}, \\
K^{\dagger} u_{j} & =\sigma_{j} v_{j}(\mathbf{r}), \\
K K^{\dagger} u_{j} & =\sigma_{j} K v_{j}(\mathbf{r})=\sigma_{i}^{2} u_{j}, \\
K^{\dagger} K v_{j}(\mathbf{r}) & =\sigma_{j} K^{\dagger} u_{j}=\sigma_{i}^{2} v_{j}(\mathbf{r}) .
\end{aligned}
$$

The inversion goal is to estimate the object function of Eqn. 35 given the measured data in $D$. We do so by expanding the object function in terms of the $v_{j}(\mathbf{r})$,

$$
\hat{o}(\mathbf{r})=\sum_{j=0}^{J-1} \alpha_{j} v_{j}(\mathbf{r}),
$$


where the $\alpha_{j}$ are constant coefficients to be determined. Substituting the object expansion into Eqn. 35, we obtain,

$$
D=\int d \mathbf{r} \Pi^{*}(\mathbf{r}) \sum_{j=0}^{J-1} \alpha_{j} v_{j}(\mathbf{r})=\sum_{j=0}^{J-1} \alpha_{j} \int d \mathbf{r} \Pi^{*}(\mathbf{r}) v_{j}(\mathbf{r}) .
$$

Applying the definition of the $K$ operator in Eqn. 36 to Eqn. 38, yields an expression for the integral of Eqn. 43,

$$
K v_{j}=\int d \mathbf{r} \Pi^{*}(\mathbf{r}) v_{j}(\mathbf{r})=\sigma_{j} u_{j}
$$

which reduces Eqn. 43 to

$$
D=\sum_{j=0}^{J-1} \alpha_{j} \sigma_{j} u_{j}
$$

Using the orthogonality of the $u_{j}$ vectors, we may solve for the unknown $\alpha_{j}$ as follows:

$$
u_{i}^{\dagger} D=\sum_{j=0}^{J-1} \alpha_{j} \sigma_{j} u_{i}^{\dagger} u_{j}=\sum_{j=0}^{J-1} \alpha_{j} \sigma_{j} \delta_{i j}=\alpha_{i} \sigma_{i},
$$

resulting in

$$
\alpha_{i}=\frac{u_{i}^{\dagger} D}{\sigma_{i}}
$$

We now require expressions for the adjoint of the forward scattering operator, $K^{\dagger}$, and the singular values and singular vectors, $\sigma_{j}, u_{j}$, and $v_{j}(\mathbf{r})$. Consider the following inner product equation which defines the adjoint,

$$
\langle u, K v\rangle=\left\langle K^{\dagger} u, v\right\rangle
$$

Using the definition of the forward scattering operator from Eqn. 36, we have

$$
u^{\dagger} \int d \mathbf{r} \Pi^{*}(\mathbf{r}) v(\mathbf{r})=\int d \mathbf{r}\left(u^{\dagger} \Pi^{*}(\mathbf{r})\right) v(\mathbf{r})
$$

Comparing the right hand sides of Eqns. 48 and 49, we obtain the following definition of the adjoint of the forward scattering operator:

$$
K^{\dagger}[\cdot] \equiv[\cdot] \cdot \Pi^{T}(\mathbf{r})
$$

The $\sigma_{j}$ and $u_{j}$ are determined by solving the eigenvalue equation of Eqn. 40 formed by the outer product of the forward scattering operator with its adjoint. Explicitly, the outer product is represented by

$$
\left(\int d \mathbf{r} \Pi^{*}(\mathbf{r}) \Pi^{T}(\mathbf{r})\right) u_{j}=\sigma_{j}^{2} u_{j}
$$


which is a $J \times J$ eigenvalue equation of the form $A x=\lambda x$. The $\Pi(\mathbf{r})$ vectors are known analytically and can be evaluated numerically. It follows that the elements of the outer product matrix can be computed numerically and the resulting system solved numerically for the $\sigma_{j}^{2}$ and $u_{j}$. Given these, we solve for $v_{j}(\mathbf{r})$ using Eqn. 39,

$$
v_{j}(\mathbf{r})=\frac{1}{\sigma_{j}} \Pi^{T}(\mathbf{r}) u_{j}
$$

Substituting Eqns. 47 and 52 into Eqn. 42, yields the final expression for the reconstruction,

$$
\hat{o}(\mathbf{r})=\sum_{j=0}^{J-1} \frac{1}{\sigma_{j}^{2}} \Pi^{T}(\mathbf{r}) u_{j} u_{j}^{\dagger} D .
$$

As described above, the $\Pi(\mathbf{r})$ vectors of Eqn. 34, and outer products and eigenvalues of Eqn. 51 are computed numerically. The system is inherently ill-conditioned due to the limited aperture of the measurement system which only measures part of the scattered field, and due to the loss of the evanescent field information. Thus, some of the eigenvalues, $\sigma_{j}^{2}$, are close to zero. Those eigenvalues and their corresponding eigenvectors determine the rank of the outer product matrix, and they must not be used in the reconstruction of Eqn. 53. A decision must be made on the number of singular values/vectors to use. We have chosen to use the best rank $N$ approximation. We compute the ratio

$$
R(N)=\frac{\sum_{j=0}^{N-1} \sigma_{j}^{2}}{\sum_{j=0}^{J-1} \sigma_{j}^{2}}
$$

where we assume the singular values are arranged from smallest to largest: $\sigma_{0}^{2} \leq \sigma_{1}^{2} \leq \cdots \leq \sigma_{J-1}^{2}$. Plotting $R(N)$, we graphically identify the point at which the function starts to rise rapidly. The index of the singular value at which this occurs, we label as $J_{0}$. With this value determined, our final reconstruction is

$$
\hat{o}(\mathbf{r})=\sum_{j=J_{0}}^{J-1} \frac{1}{\sigma_{j}^{2}} \Pi^{T}(\mathbf{r}) u_{j} u_{j}^{\dagger} D .
$$

Our experience reconstructing both simulated and experimentally collected scattered field data have shown that this criterion works consistently well.

The HSIW is extremely flexible in that it allows any transducer configuration and any number of frequencies to be used in forming the reconstruction. The disadvantage is the potentially large amount of computer resources required for processing. Computing the outer product of Eqn. 51 and its eigenvalues is time consuming for high resolution reconstructions. Short term disk storage space for the $\Pi(\mathbf{r})$ vectors of Eqn. 34 was approximately $2 \mathrm{~Gb}$ for the reconstructions of Section 7.

In the next section, we present the results of a proof-of-principle example based upon the exact Born approximation scattering model of Eqn. 8. 


\section{Proof-of-Principle}

Our proof-of-principle consists of two radial reflection simulations run under the Born approximation of Eqn. 8. The geometry, shown in Figure 4, consists of five point scatterers at a fixed radius but with increasing angular separation so as to achieve an angular resolution test. The first simulation was performed using a multimonostatic operating mode with 84 transducers and 100 reconstruction frequencies, the second used a multistatic operating mode with 20 sources co-located with 20 receivers and 21 frequencies. These numbers were selected so that in both cases the number of elements, $J$, in the projection kernel of Eqn. 34, is 8400. This was done in order to equalize the comparison of the reconstruction results and minimize algorithmic differences. We did not model any shadowing due to the probe. That is, fields launched and/or received by transducers 180 degrees away from a scatterer were able to propagated to/from the scatterer. The forward scattered field is computed via

$$
\psi_{B}^{s c a t}\left(\mathbf{R}_{m}^{r}, \mathbf{R}_{n}^{t}, \omega_{l}\right)=P\left(\omega_{l}\right) k_{0}^{2}\left(\omega_{l}\right) \sum_{s=0}^{S-1} G\left(\mathbf{R}_{m}^{r}, \mathbf{X}_{s}, \omega_{l}\right) G\left(\mathbf{X}_{s}, \mathbf{R}_{n}^{t}, \omega_{l}\right),
$$

where $S$ is the number of point scatterers, $\mathbf{X}_{s}$ is the location of the $s$-th scatterer, and $G\left(\mathbf{r}, \mathbf{r}^{\prime}, \omega\right) \equiv$ $(i / 4) H_{0}^{(1)}\left(k_{0}(\omega)\left|\mathbf{r}-\mathbf{r}^{\prime}\right|\right)$ is the two-dimensional Green function.

The incident pulse used in the simulation is described by the first derivative of a Gaussian, or "DOG" pulse, given by

$$
p(t)=-e^{1 / 2} \omega_{0}\left(t-t_{0}\right) e^{-\left(\omega_{0}\left(t-t_{0}\right)\right)^{2} / 2},
$$

where $\omega_{0} \equiv 2 \pi f_{0}$ and $f_{0}$ is the peak frequency of the pulse in Hertz. The pulse is presented in Figure 5(a). Figure 5(b) and (c) show the pulse spectrum with the frequencies used in the multimonostatic and multistatic reconstructions, respectively. Figure 6 shows the singular value ratio, $R(N)$, of Eqn. 54. The selection of the number of singular values to use was performed manually and the threshold is shown in the figure. The multimonostatic reconstruction used 5880 of the 8400 eigenvalues (70\%), and the multistatic required $2100(25 \%)$.

The reconstructions are shown in Figure 7. The multimonostatic reconstruction reveals better resolution and somewhat better contrast over the multistatic reconstruction. This reflects the greater number of frequencies used in the reconstruction. As shown in Figure 3, the more frequencies used results in a larger area in the object's spatial spectrum being reconstructed. For point scatterers, the multimonostatic operating mode is sufficient. The results of the next section demonstrate that this mode is insufficient for imaging extended scatterers under realistic wave propagation conditions.

\section{Full Wave Simulation}

We performed a two-dimensional finite-difference time-domain (FDTD) simulation of wave propagation in a domain similar to that of the proof-of-principle example. We used E3D, an explicit 2D/3D elastic/acoustic propagation code designed for modeling seismic waves, developed 
at Lawrence Livermore National Laboratory $[6,7,8,9,10,11]$. The code simulates full wave scattering and requires as inputs a longitudinal velocity distribution, a shear velocity distribution, and a density distribution. In this case, unlike the proof-of-principle, the probe was included in the simulation, shadowing fields from transducers and scatterers with large angular separation.

The FDTD simulation domains are shown in Figure 8. The domains consist of a homogeneous background with two elliptical inclusions. One inclusion's longitudinal velocity and density are $15 \%$ above the background; the other's are 5\% below. There is no variation in shear velocity. The dashed line indicates the transducer outline.

As in the proof-of-principle example, we performed both a multimonostatic and multistatic simulation. The former used 50 transducer locations with 120 reconstruction frequencies resulting in $J=50 \times 120=6000$ elements in the forward propagation kernel. The latter used 50 source locations and a receiving aperture of 15 transducers centered about the active source. As the transmitting element rotated, so did the receiving aperture, as indicated in Figure 1(c). The exact multistatic configuration for this simulation is shown in Figure 9. The multistatic reconstruction used 8 frequencies to achieve a $J=50 \times 15 \times 8=6000$ element kernel. As in the proofof-principle example, we used kernels with identical number of elements to minimize variability when comparing the quality of the reconstructions. The simulation configurations and parameters are listed in the first two lines of Table 1.

The full wave simulations used the same "DOG" pulse as the proof-of-principle example. The frequencies used in the reconstruction for each of the two cases are presented in Figure 10. Again, we used the best rank $N$ approximation to determine the number of singular values and vectors to use in the reconstructions. The singular value ratio, $R(N)$, and the singular value distribution are plotted in Figure 11. The multimonostatic case required $4260(71 \%)$ of the singular values, and the multistatic required 1020 (17\%). The magnitude of the reconstructions are shown in Figure 12. The high-pass nature of reflection mode diffraction tomography as represented in Figure 3 is demonstrated in the multimonostatic reconstruction of Figure 12 (a) where the edges of the scattering objects are clearly highlighted. There is, however, an azimuthal ambiguity resulting from the use of only one transducer: off radial axis scattering can only be resolved to within a fixed range from the transducer. Thus, off radial axis scattering limits the usefulness of multimonostatic operation. Better azimuthal localization is achieved when more receiving transducers are included, widening the agular receiving aperture, permitting the resolution of the azimuthal ambiguity. This is demonstrated in the multistatic reconstruction of Figure 12(b). Observe however, when fewer frequencies are uses, range resolution is lost.

We studied the receiver number/reconstruction frequency trade-off further in the three other multistatic simulations listed in Table 1. We decreased the number of receivers and increased the number of reconstruction frequencies while keeping the size of the problem within our computer resources. The reconstructions are shown in Figure 13. We confirm that scatterer azimuthal localization improves with the number of receivers but there is a loss of radial resolution when fewer frequencies are used. We conclude that azimuthal resolution is achieved by increasing the number of receivers, where as range resolution increases with the number of frequencies. 


\section{Conclusions}

We set out to develop a wave-based tomographic imaging tool based upon an intravascular ultrasound probe model in which a single rotating transducer both insonofies the surrounding medium and measured the backscattered field. Our goal was to develop a wave-based inversion algorithm to image the surrounding medium rather than using the standard B-scan currently used that are based upon straight ray sound propagation.

We developed an analytic inverse for the multimonostatic configuration but were unable to implement it numerically. We then implemented a purely numerical inverse based upon the new Hilbert space inverse wave (HSIW) algorithm. The flexibility of the HSIW is such that it allows any configuration of transducers and frequencies to be used in the reconstruction. This permitted us to test not only a multimonostatic operating mode but also various multistatic modes.

We concluded that the multimonostatic mode lacks sufficient spatial diversity to image correctly scattering targets, and that scatterer azimuthal localization improves as more receivers are used and that range resolution improves as more frequencies are used.

\section{A Relationship Between the Fourier \& Fourier-Bessel Trans- form}

We wish to relate the Fourier-Bessel Diffraction Theorem of Eqn. 27 to the Fourier Diffraction Theorem so that we may understand how the object's spatial spectrum is covered by the radial reflection measurement system. In order to do so, we require a relation between the FourierBessel transform of the object to its Fourier transform. We consider a two dimensional function represented in Cartesian coordinates as $f(x, y)$ or in polar coordinates as $f(r, \theta)$ where we have the following relationship between the coordinate pairs,

$$
\begin{aligned}
& x=r \cos \theta, \\
& y=r \sin \theta .
\end{aligned}
$$

The Fourier-Bessel transform pair are

$$
\begin{aligned}
& F_{m}(k)=\frac{1}{2 \pi} \int_{0}^{2 \pi} d \theta \int_{0}^{\infty} r d r f(r, \theta) e^{-i m \theta} J_{m}(k r), \\
& f(r, \theta)=\sum_{m=-\infty}^{\infty} \int_{0}^{\infty} k d k F_{m}(k) e^{i m \theta} J_{m}(k r),
\end{aligned}
$$

and the Cartesian Fourier transform pair are,

$$
\begin{aligned}
F\left(k_{x}, k_{y}\right) & =\int_{-\infty}^{\infty} d x \int_{-\infty}^{\infty} d y F(x, y) e^{-i\left(k_{x} x+k_{y} y\right)} \\
f(x, y) & =\frac{1}{(2 \pi)^{2}} \int_{-\infty}^{\infty} d k_{x} \int_{-\infty}^{\infty} d k_{y} F\left(k_{x}, k_{y}\right) e^{i\left(k_{x} x+k_{y} y\right)}
\end{aligned}
$$


The Cartesian Fourier transform pair are cast into polar coordinates using the following change of variables,

$$
\begin{aligned}
& k_{x}=k \cos \phi, \\
& k_{y}=k \sin \phi,
\end{aligned}
$$

to yield,

$$
\begin{aligned}
F(k, \phi) & =\int_{0}^{2 \pi} d \theta \int_{0}^{\infty} r d r f(r, \theta) e^{-i k r \cos (\theta-\phi)}, \\
f(r, \theta) & =\frac{1}{(2 \pi)^{2}} \int_{0}^{2 \pi} d \phi \int_{0}^{\infty} k d k F(k, \phi) e^{i k r \cos (\theta-\phi)} .
\end{aligned}
$$

To determine the relationship between $F(k, \phi)$ and $F_{m}(k)$, we substitute the expansion

$$
e^{-i k r \cos \theta}=\sum_{m=-\infty}^{\infty}(-i)^{m} J_{m}(k r) e^{i m \theta}
$$

into Eqn. 64 to find

$$
F(k, \phi)=\sum_{m=-\infty}^{\infty}(-i)^{m} e^{i m \phi} F_{m}(k)
$$

Using $\frac{1}{2 \pi} \int_{0}^{2 \pi} d \theta e^{i(m-n) \theta}=\delta_{m n}$, we may invert Eqn. 66 to find

$$
F_{m}(k)=\frac{(-i)^{-m}}{2 \pi} \int_{0}^{2 \pi} d \phi F(k, \phi) e^{-i m \phi} .
$$




\begin{tabular}{|c|c|c|c|c|c|}
\hline $\begin{array}{c}\text { Simulation } \\
\text { Configuration }\end{array}$ & $\begin{array}{c}\text { Number of } \\
\text { Source } \\
\text { Locations }\end{array}$ & $\begin{array}{c}\text { Number of } \\
\text { Receivers }\end{array}$ & $\begin{array}{c}\text { Aperture } \\
\text { Angle }\end{array}$ & $\begin{array}{c}\text { Number of } \\
\text { Reconstruction } \\
\text { Frequencies }\end{array}$ & $\begin{array}{c}\text { Number of } \\
\text { HSIW Kernel } \\
\text { Elements, } J\end{array}$ \\
\hline Multimonostatic & 50 & 1 & $\mathrm{n} / \mathrm{a}$ & 120 & 6000 \\
Multistatic & 50 & 15 & $100.8^{\circ}$ & 8 & 6000 \\
Multistatic & 50 & 15 & $100.8^{\circ}$ & 12 & 9000 \\
Multistatic & 50 & 9 & $57.6^{\circ}$ & 21 & 9450 \\
Multistatic & 50 & 5 & $28.8^{\circ}$ & 37 & 9520 \\
\hline
\end{tabular}

Table 1: FDTD simulation configuration and reconstruction parameters. 


\section{Multimonostatic}

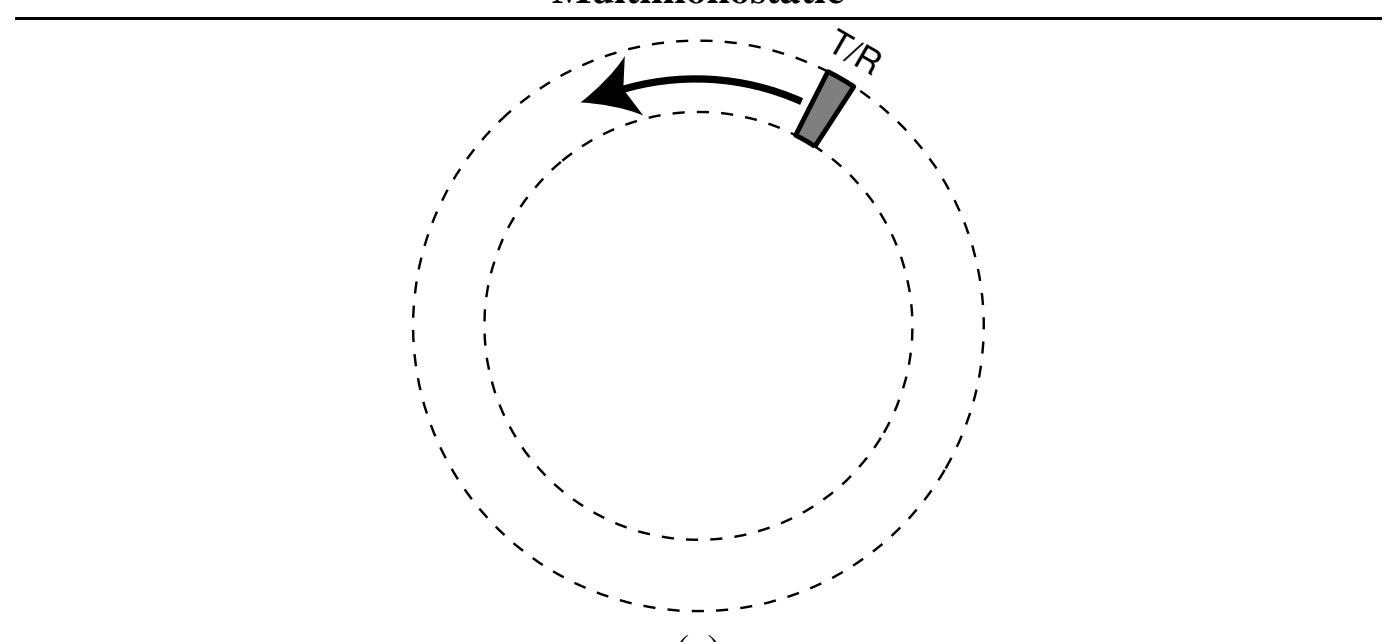

(a)

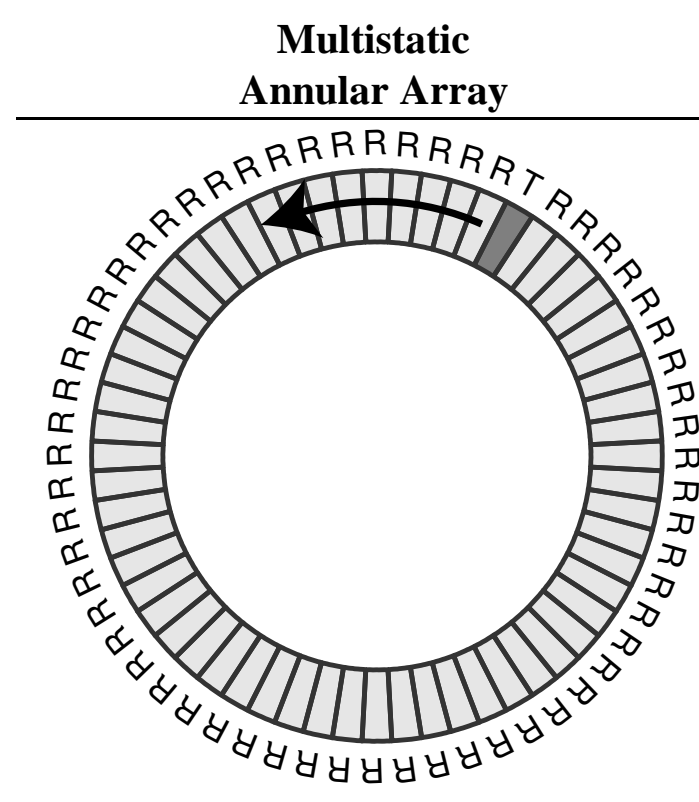

(b)

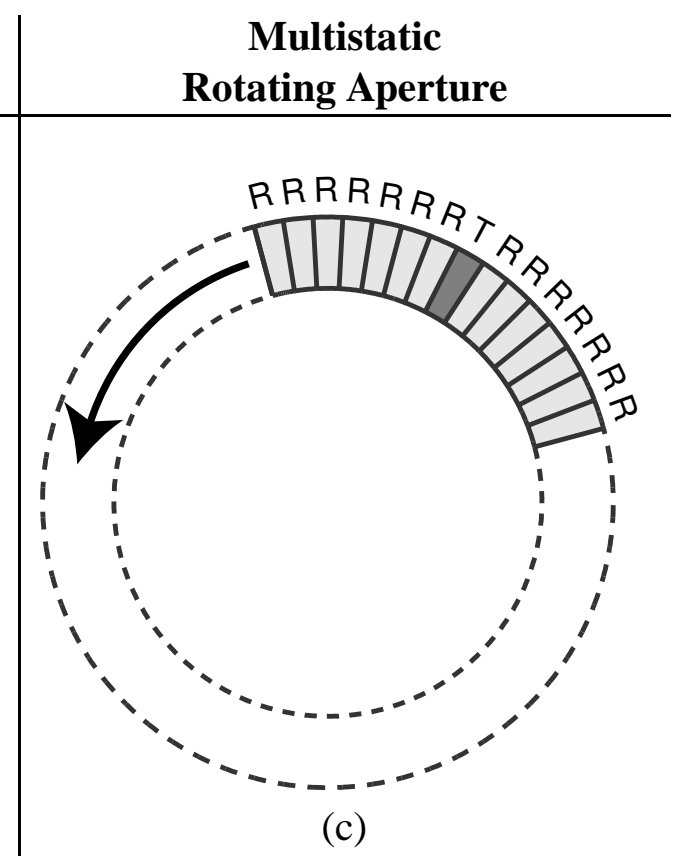

(c)

Figure 1: Three radial reflection transducer configurations. (a) The multimonostatic mode: a single transducer rotates about a fixed center. At each angular location it launches the primary field and collects the reflected scattered field. (b) A multistatic case consisting of a fixed annular array of outward looking transducers. In succession, each transducer launches the primary field and the reflected scattered field is measured at all the transducers. (c) A multistatic configuration consisting of a rotating aperture. 


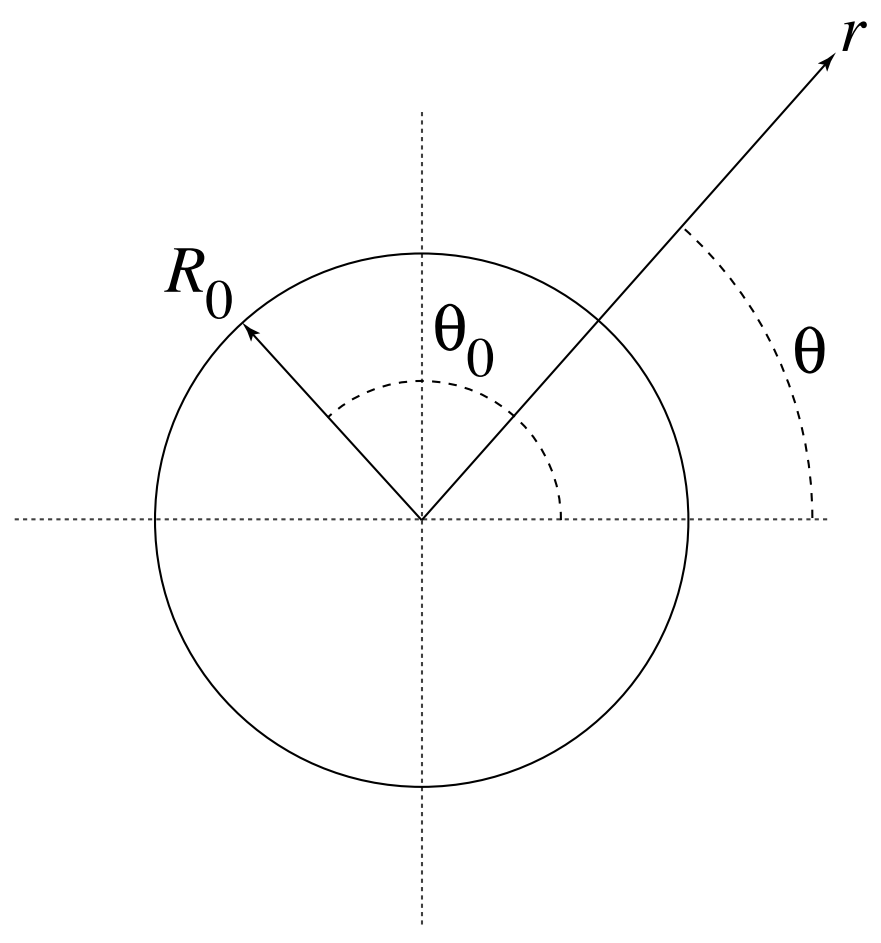

Figure 2: Radial reflection geometry. $R_{0}$ is the probe diameter, $\mathbf{r}_{0} \equiv R_{0}\left(\cos \theta_{0}, \sin \theta_{0}\right)$ is the planar location of the transducer, $\mathbf{r} \equiv r(\cos \theta, \sin \theta)$ is a point within the surrounding medium. 


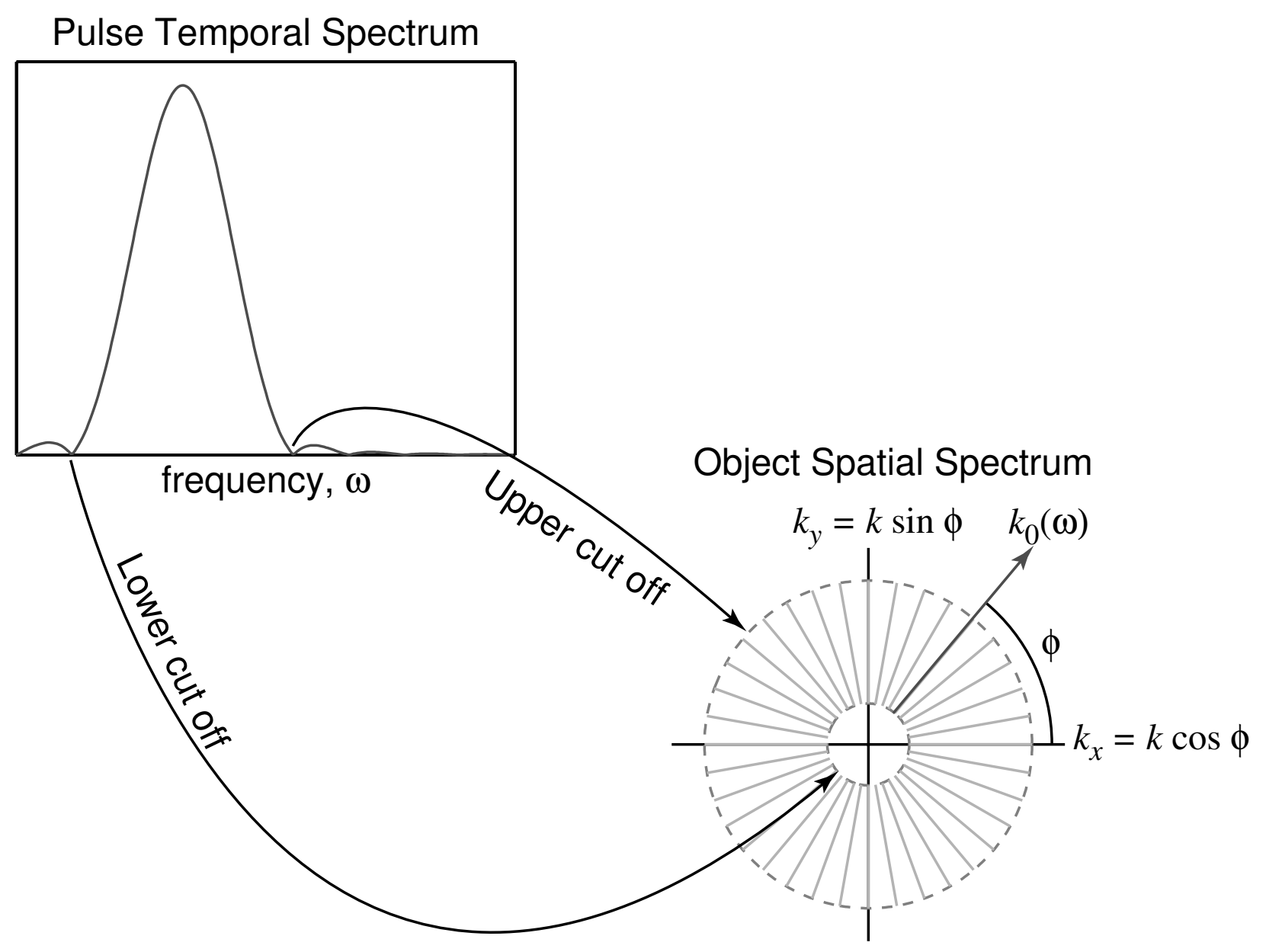

Figure 3: Radial reflection Fourier diffraction theorem. 


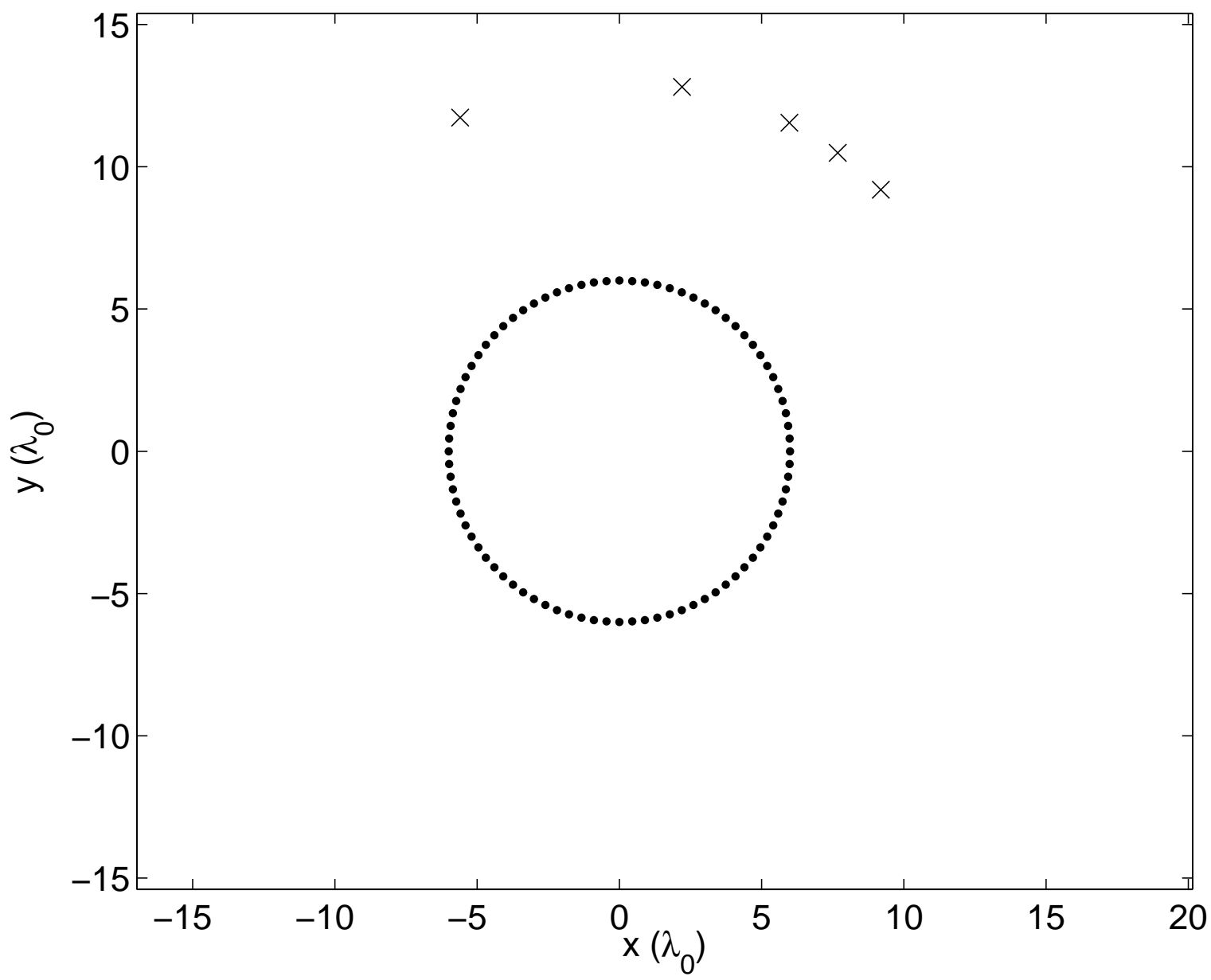

Figure 4: Proof-of-principle geometry. Two simulations were performed: a multimonostatic run with 84 transducers which used 100 frequencies for the reconstruction, and a multistatic run with 20 transducers which used 21 frequencies. 


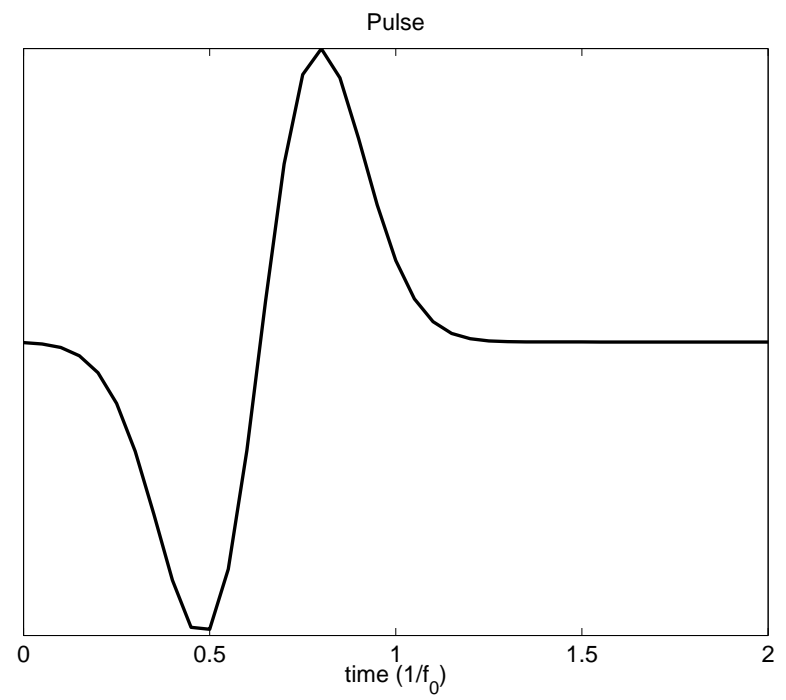

(a)

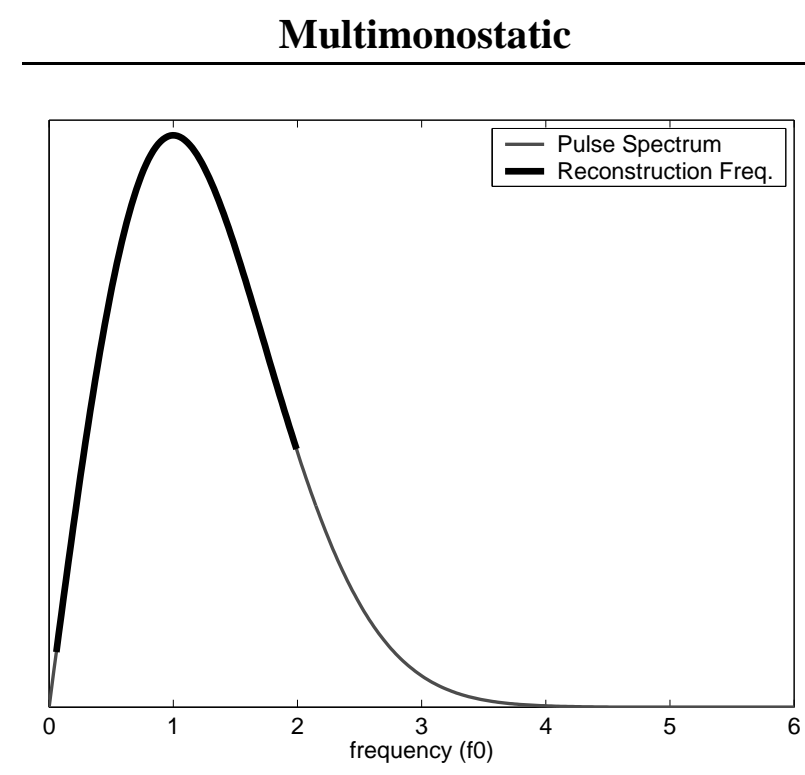

(b)
Multistatic

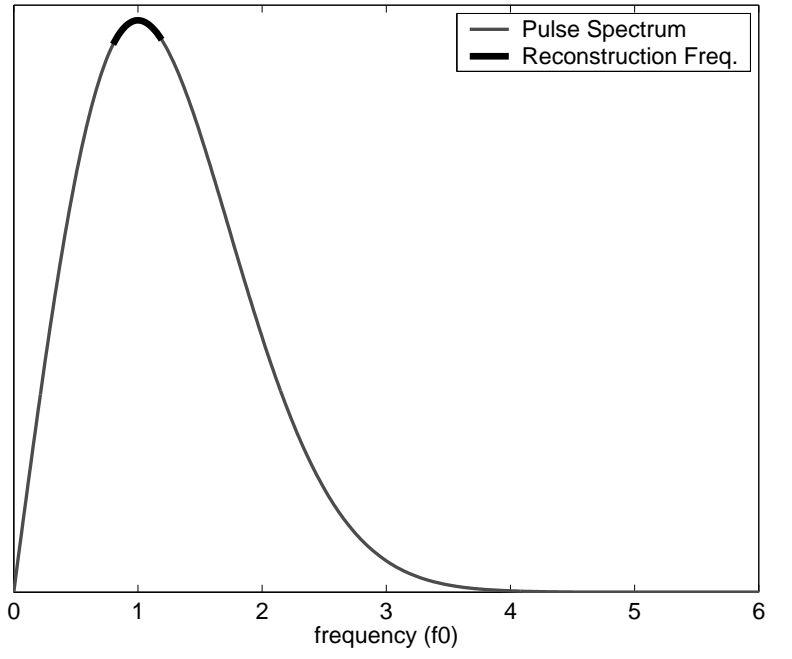

(c)

Figure 5: Proof-of-principle pulse. (a) Time domain derivative of a Gaussian (DOG) pulse. (b) DOG pulse spectrum with the 100 frequencies used in the multimonostatic reconstruction highlighted. (c) DOG pulse spectrum with the 21 frequencies used in the multistatic reconstruction. 

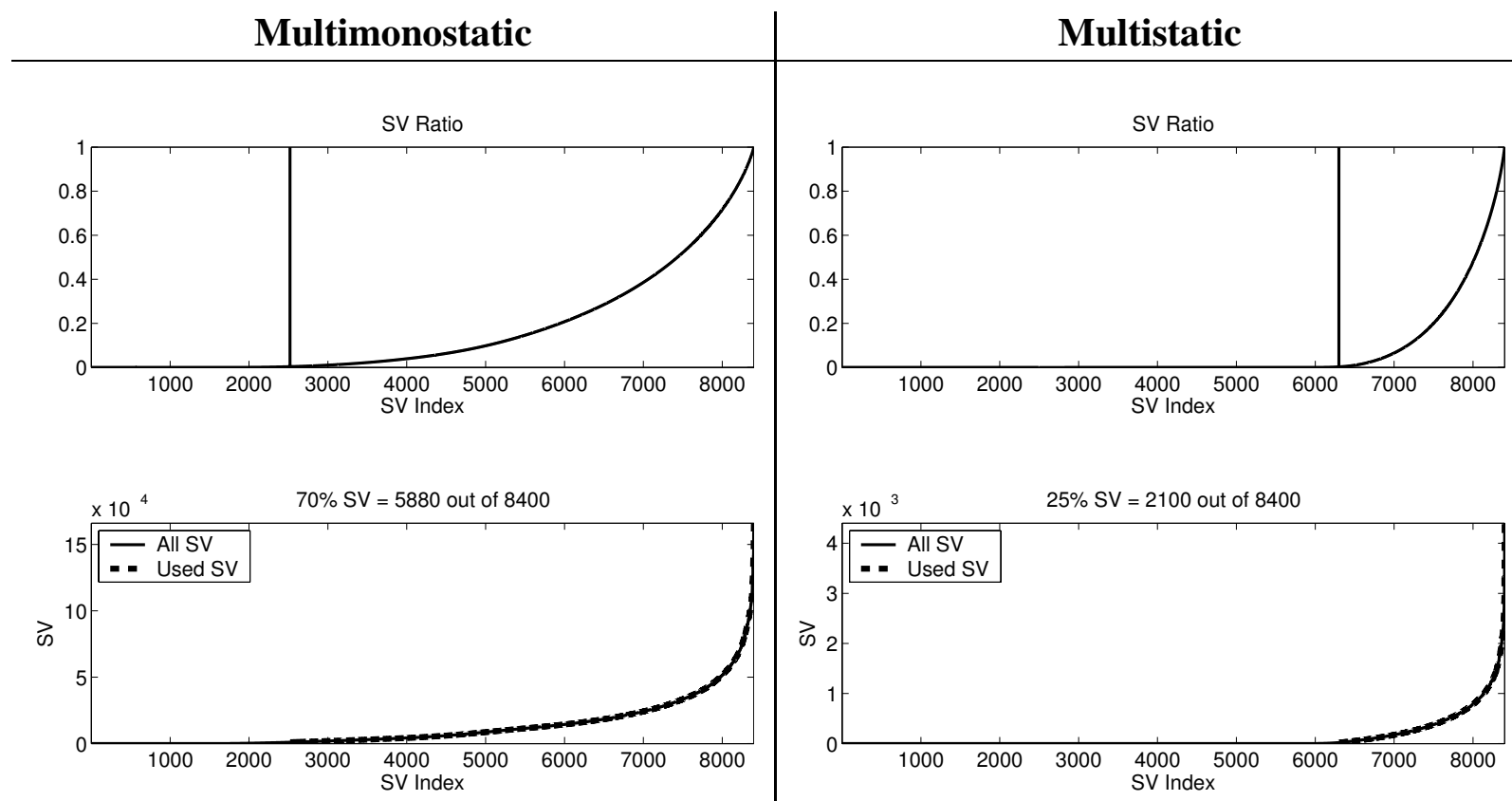

(a)

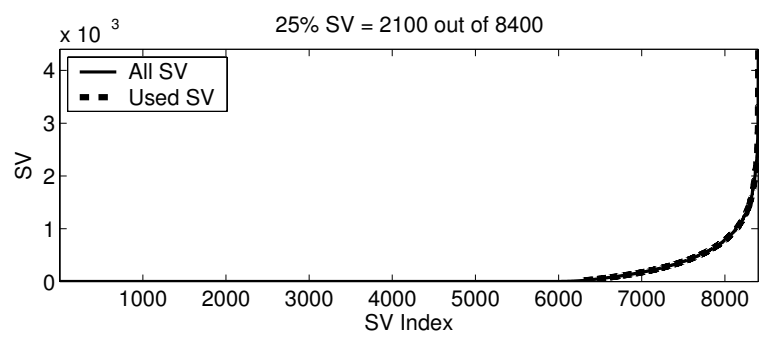

(b)

Figure 6: Proof-of-principle singular values. The top plots show the eigenvalue ratios, $R(N)$, of Eqn. 54. The vertical line shows where the selection was made "zero" and non-zero singular values. The bottom plots show the actual singular values. The highlighted shows those singular values used in the reconstructions. The (a) column is the multimonostatic case where $70 \%$ or 5880 out of 8400 singular values/vectors were used; the (b) column is the multistatic case where $25 \%$ or 2100 singular values/vectors were used. 


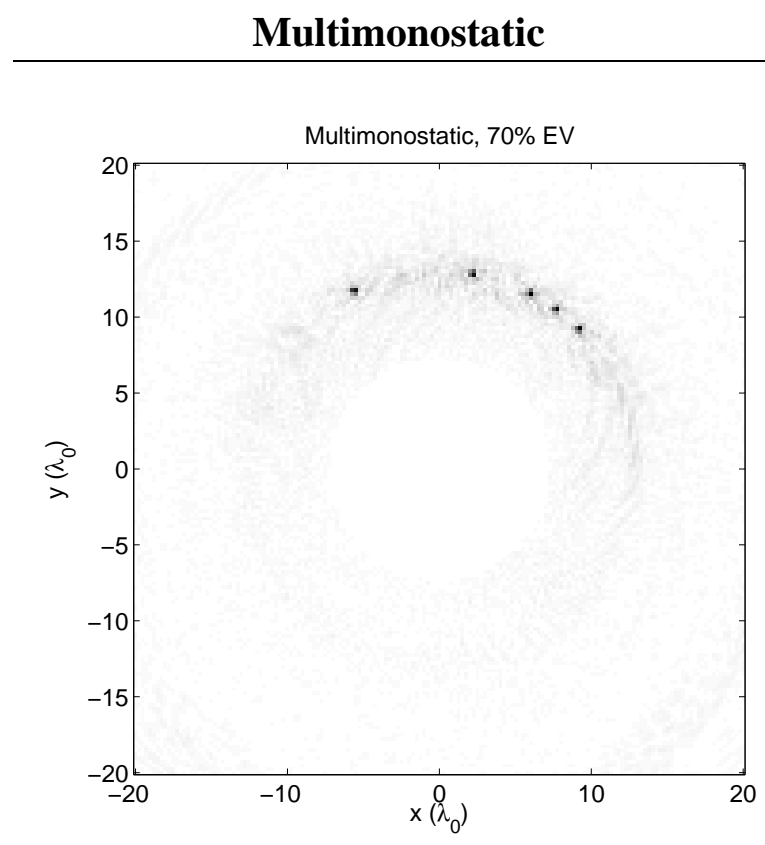

(a)
Multistatic

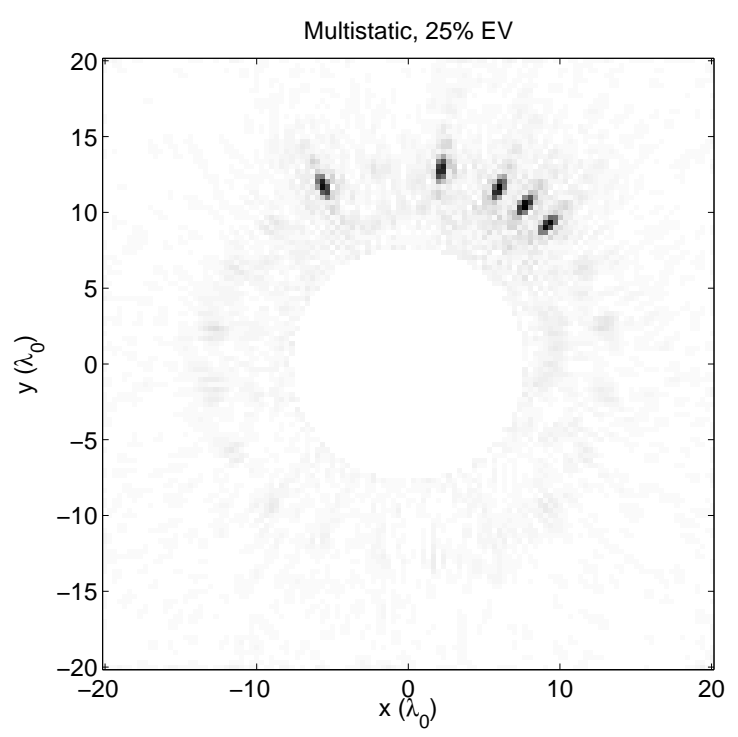

(b)

Slices through Reconstructions

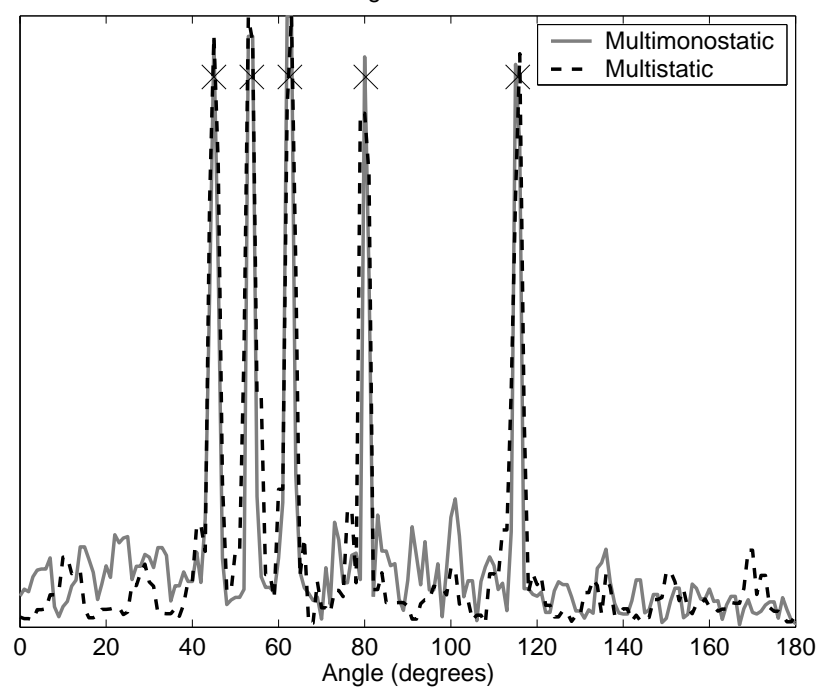

(c)

Figure 7: Proof-of-principle reconstructions. (a) Multimonostatic. (b) Multistatic. (c) Angular slices through the reconstructions at the radius of the scatterers. The $\times$ indicate the actual location of the scatterers. The multimonostatic case shows better resolution and somewhat higher contrast than the multistatic case. This is a consequence of the greater number of frequencies used in the reconstruction which increased the coverage in the object's spatial spectrum. 

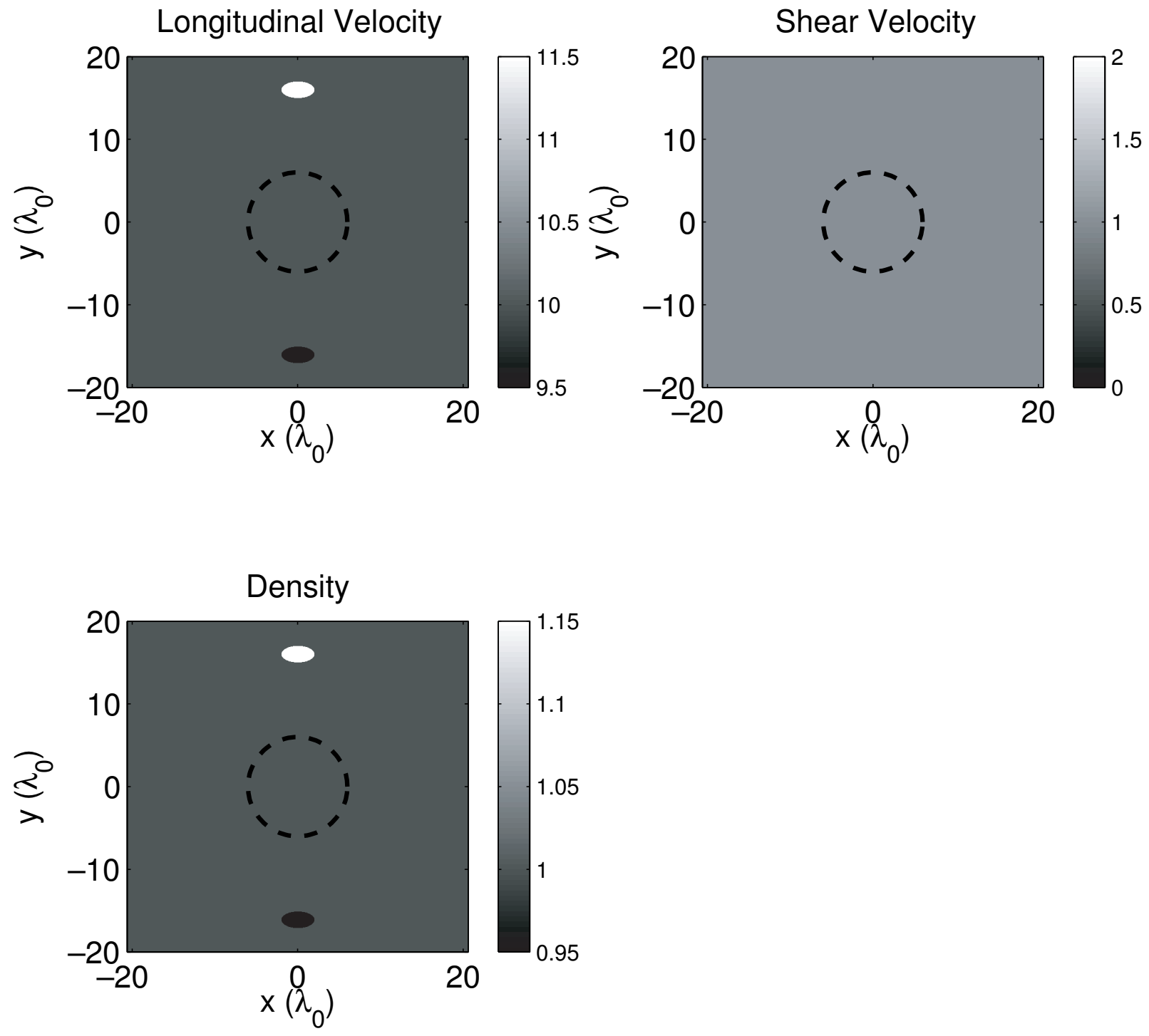

Figure 8: Finite-difference time-domain simulation domains. The domains consist of a homogeneous background with two elliptical inclusions. One inclusion's longitudinal velocity and density are $15 \%$ above the background; the other's are 5\% below. There is no variation in shear velocity. The dashed line indicates the transducer outline. 


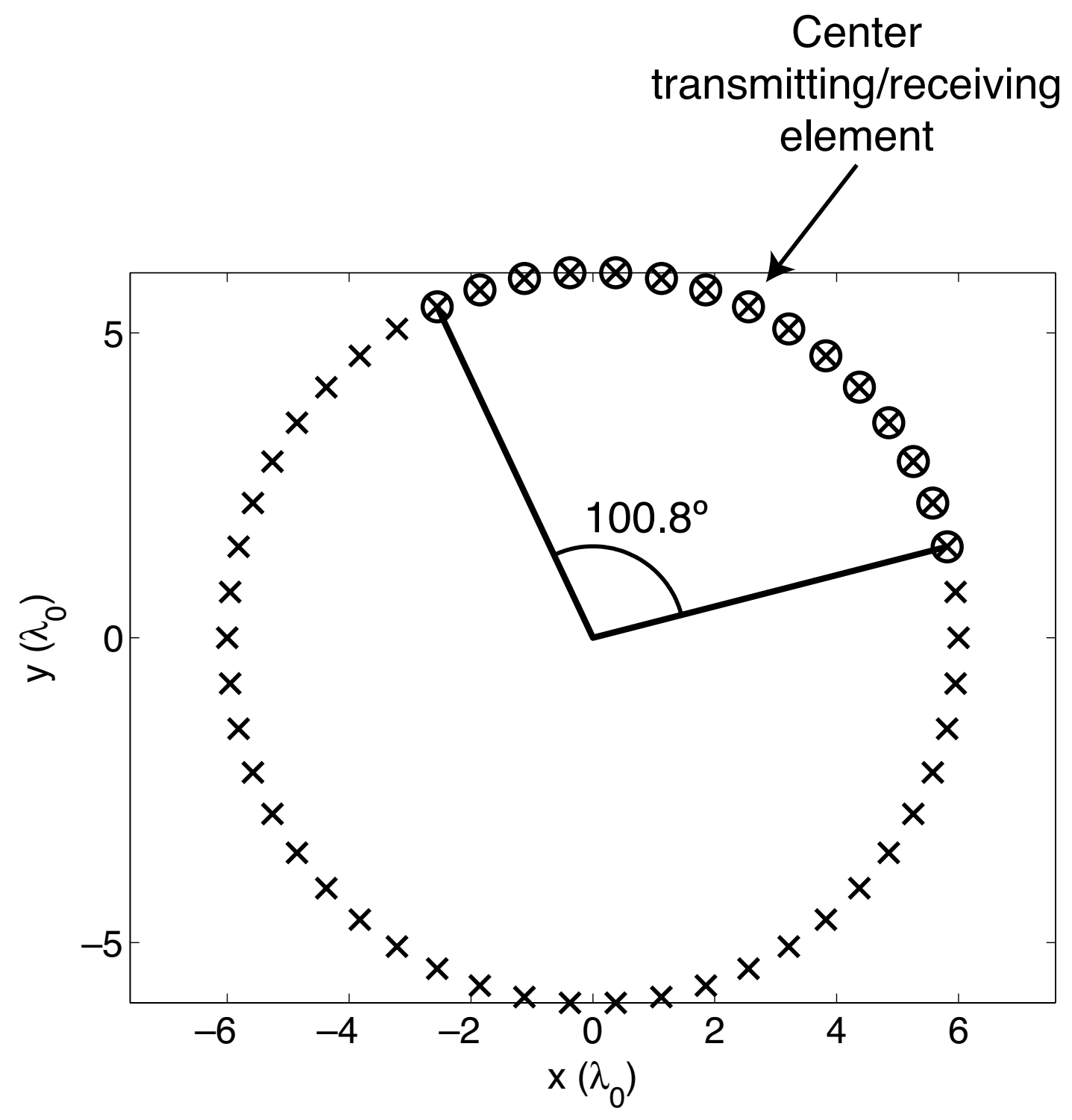

Figure 9: The transducer geometry for the FDTD simulations. The $\times$ mark the 50 sourc positions. The circles indicate the active receivers within the rotating sub-aperture. For the 15-element aperture, the aperture angle is $100.8^{\circ}$. 


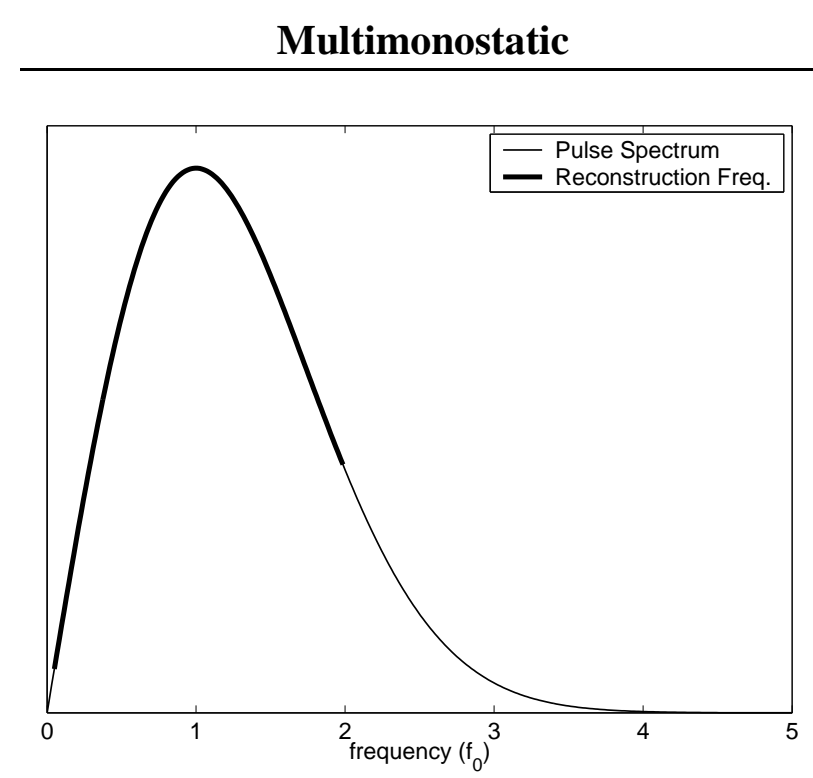

(b)
Multistatic

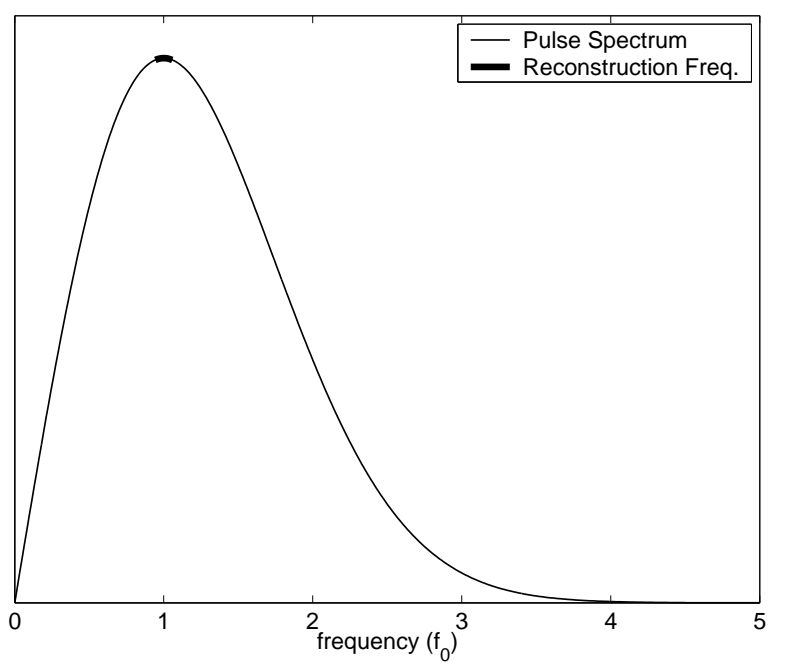

(c)

Figure 10: FDTD pulse spectrum. (a) DOG pulse spectrum with the 120 frequencies used in the multimonostatic reconstruction highlighted. (b) DOG pulse spectrum with the 8 frequencies used in the multistatic reconstruction. 

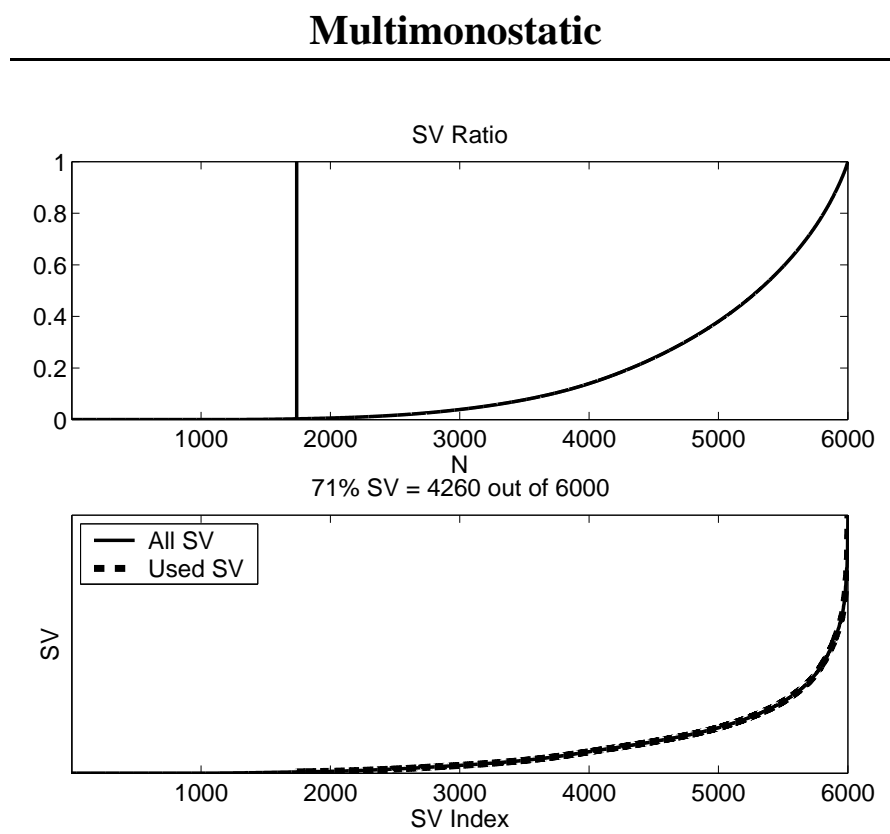

(a)
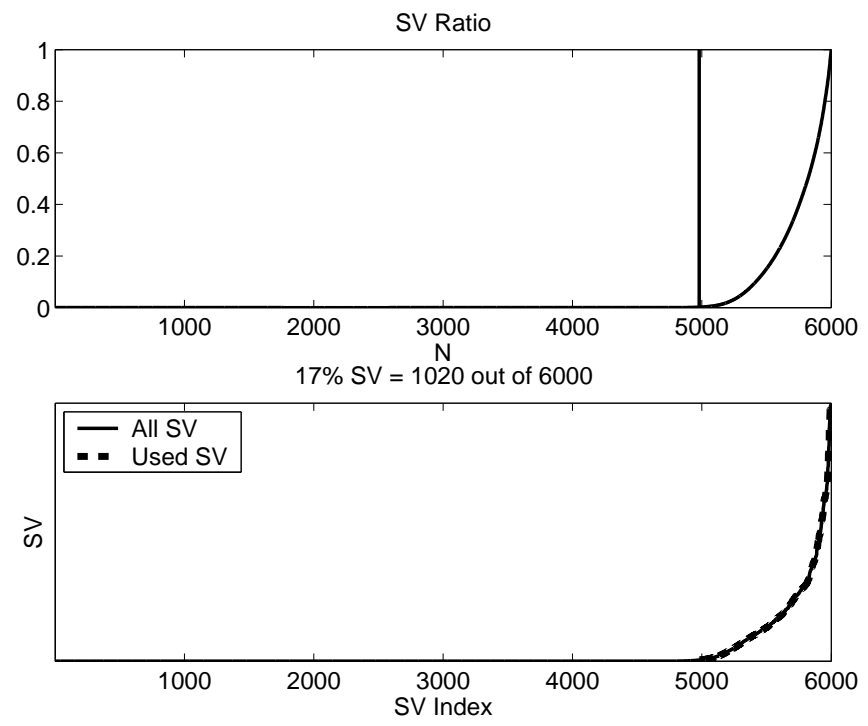

(b)

Figure 11: FDTD simulation singular values. The top plots show the singular values ratios, $R(N)$, of Eqn. 54. The vertical line shows where the selection was made distinguishing between the "zero" and non-zero singular values, thus determining the rank of the system. The bottom plots show the actual singular values. The highlighted trace shows those singular values used in the reconstructions. The (a) column is the multimonostatic case, the (b) column is the multistatic case. 


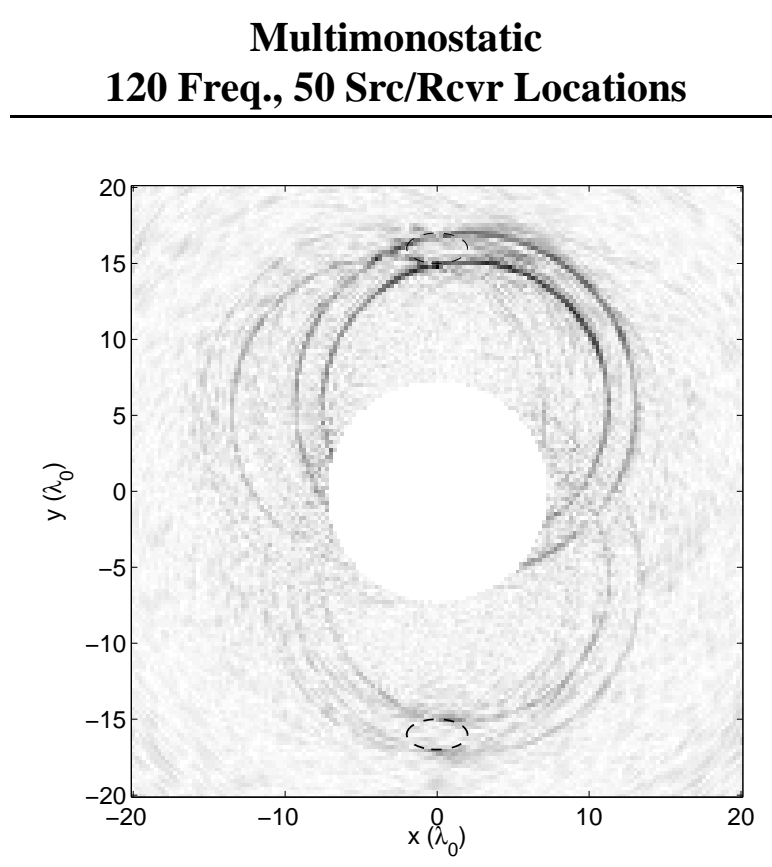

(a)

\section{Multistatic \\ 8 Freq., 50 Src Locations, 15 Receivers}

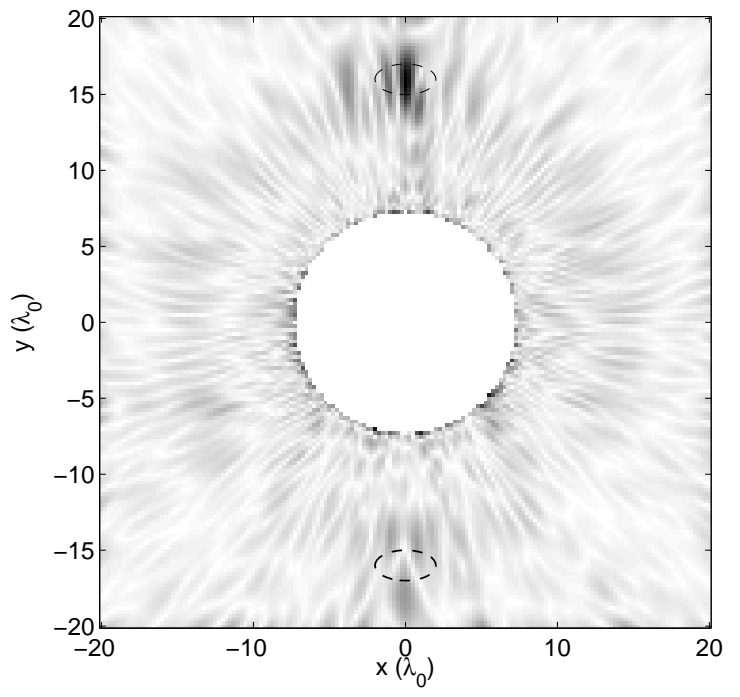

(b)

Figure 12: Reconstructions of the FDTD simulations. (a) Multimonostatic using $71 \%$ of the singular values. (b) Multistatic using $17 \%$ of the singular values. 


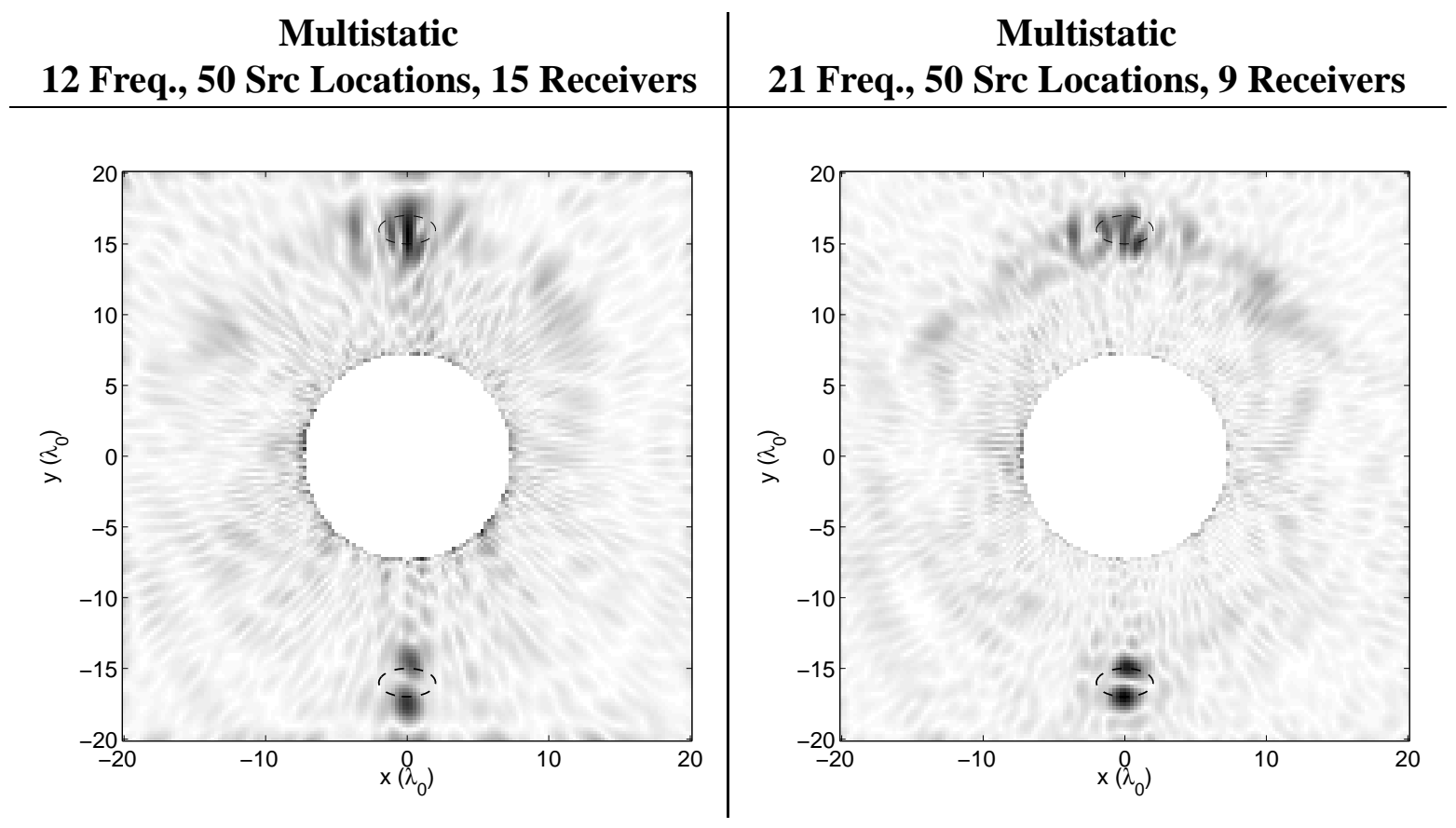

Multistatic

37 Freq., 50 Src Locations, 5 Receivers

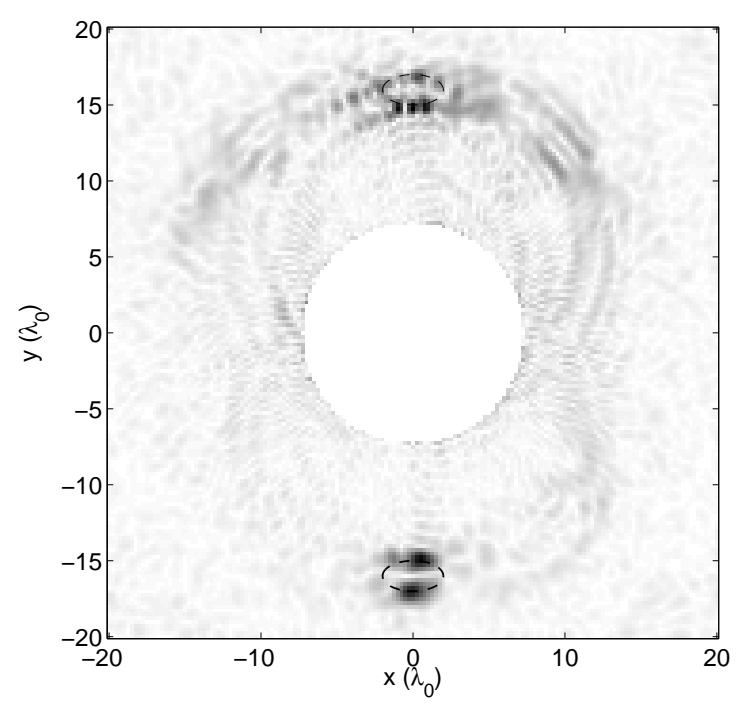

Figure 13: Additional multistatic reconstructions to evaluate the trade-off between receiving aperture size and the number of frequencies used in the reconstruction. Radial resolution increases with the number of frequencies used, and azimuthal resolution increases aperture size. 


\section{References}

[1] A. J. Devaney. A filtered backpropagation algorithm for diffraction tomography. Ultrasonic Imaging, 4(4):336-350, October 1982.

[2] A. C. Kak and M. Slaney. Principles of Computerized Tomographic Imaging. IEEE Press, 1988. ISBN 0-7803-0447-0.

[3] K. J. Langenberg. Applied inverse problems for acoustic, electromagnetic and elastic wave scattering. In P. C. Sabatier, editor, Basic Methods of Tomography and Inverse Problems, Malvern Physics Series, chapter II, pages 125-467. Adam Hilger, 1987. A set of lectures by G. T. Herman, H. K. Tuy, K. J. Langenberg, and P. C. Sabatier. ISBN 0-85274-285-1.

[4] P. G. Yock, P. J. Fitzgerals, and Y. Honda. Intravascular Ultrasound. In E. J. Topol, editor, Textbook of Interventional Cardiology. Saunders, 1999.

[5] A. J. Devaney and M. Dennison. Inverse scattering in inhomogeneous background media. Inverse Problems, 19:855-870, 2003.

[6] A. R. Levander. Fourth-order finite-difference p-sv seismograms. Geophysics, 53:14251436, 1988.

[7] S. Larsen and J. Grieger. Elastic modeling initiative, part iii: 3-d computational modeling. Soc. Expl. Geophysi. Confer. Proc., 68:1803-1806, 1998.

[8] S. Larsen, M. Antolik, and et. al. 3d simulations of scenario earthquakes in the San Francisco Bay area. EOS Trans., 78:487, 1997.

[9] R. Madariaga. Dynamics of an expanding circular fault. Bulletin of the Seismological Society of America, 66(3):639-666, June 1976.

[10] J. Virieux. P-SV wave propagation in heterogeneous media: Velocity-stress finite-difference method. Geophysics, 51(4):889-901, April 1986.

[11] Shawn Larsen and David Harris. Seismic wave propagation through a low-velocity nuclear rubble zone. Technical report, Lawrence Livermore National Laboratory, 7000 East Avenue, Livermore, CA 94550, 1993.

[12] G. Barton. Elements of Green's Functions and Propagation - Potentials, Diffusion and Waves. Oxford University Press, 1991. ISBN 0-19-851988-6.

[13] S. J. Norton and M. Linzer. Ultrasonic reflectivity imaging in three dimensions: Exact inverse scattering solutions for plane, cylindrical, and spherical apertures. IEEE Transactions on Biomedical Engineering, BME-28(2):202-220, February 1981. 